Article

\title{
Exploring the Chemodiversity and Biological Activities of the Secondary Metabolites from the Marine Fungus Neosartorya pseudofischeri
}

\section{Wan-Ling Liang ${ }^{1,2}$, Xiu Le ${ }^{1}$, Hou-Jin Li ${ }^{3}$, Xiang-Ling Yang ${ }^{4,5,6}$, Jun-Xiong Chen ${ }^{4,5,6}$, Jun Xu ${ }^{1}$, Huan-Liang Liu ${ }^{4,5,6}$, Lai-You Wang ${ }^{7}$, Kun-Teng Wang ${ }^{7}$, Kun-Chao Hu ${ }^{1,2}$, De-Po Yang ${ }^{1,2}$ and Wen-Jian Lan ${ }^{1,2, *}$}

1 School of Pharmaceutical Sciences, Sun Yat-sen University, Guangzhou 510006, China; E-Mails: natprodlwl@gmail.com (W.-L.L.); lexiu2012@163.com (X.L.); junxu@biochemomes.com (J.X.); kunchao89@gmail.com (K.-C.H.); 1ssydp@mail.sysu.edu.cn (D.-P.Y.)

2 Guangdong Technology Research Center for Advanced Chinese Medicine, Guangzhou 510006, China

3 School of Chemistry and Chemical Engineering, Sun Yat-sen University, Guangzhou 510275, China; E-Mail: ceslhj@mail.sysu.edu.cn

4 Guangdong Institute of Gastroenterology, Guangzhou 510655, China; E-Mails: morningyang100@gmail.com (X.-L.Y.); junxiong_ch@sina.com (J.-X.C.); huanliang.liu@gmail.com (H.-L.L.)

5 Guangdong Key Laboratory of Colorectal and Pelvic Floor Diseases, Guangzhou 510655, China

6 The Sixth Affiliated Hospital, Sun Yat-sen University, Guangzhou 510655, China

7 Institute of Chinese Medical Sciences, Guangdong Pharmaceutical University, Guangzhou 510006, China; E-Mails: wanglaiyou@gdpu.edu.cn (L.-Y.W.); kuntengwang@gmail.com (K.-T.W.)

* Author to whom correspondence should be addressed; E-Mail: lanwj@mail.sysu.edu.cn; Tel.: +86-20-3994-3042; Fax: +86-20-3994-3000.

External Editor: Orazio Taglialatela-Scafati

Received: 15 August 2014; in revised form: 12 November 2014 / Accepted: 17 November 2014 / Published: 24 November 2014

\footnotetext{
Abstract: The production of fungal metabolites can be remarkably influenced by various cultivation parameters. To explore the biosynthetic potentials of the marine fungus, Neosartorya pseudofischeri, which was isolated from the inner tissue of starfish Acanthaster planci, glycerol-peptone-yeast extract (GlyPY) and glucose-peptone-yeast extract (GluPY) media were used to culture this fungus. When cultured in GlyPY medium,
} 
this fungus produced two novel diketopiperazines, neosartins $\mathrm{A}$ and $\mathrm{B}$ (1 and 2), together with six biogenetically-related known diketopiperazines, 1,2,3,4-tetrahydro-2, 3-dimethyl-1,4-dioxopyrazino[1,2-a]indole (3), 1,2,3,4-tetrahydro-2-methyl-3-methylen e-1,4-dioxopyrazino[1,2-a]indole (4), 1,2,3,4-tetrahydro-2-methyl-1,3,4-trioxopyrazino[1,2-a] indole (5), 6-acetylbis(methylthio)gliotoxin (10), bisdethiobis(methylthio)gliotoxin (11), didehydrobisdethiobis(methylthio)gliotoxin (12) and $\mathrm{N}$-methyl- $1 \mathrm{H}$-indole-2-carboxamide (6). However, a novel tetracyclic-fused alkaloid, neosartin C (14), a meroterpenoid, pyripyropene A (15), gliotoxin (7) and five known gliotoxin analogues, acetylgliotoxin (8), reduced gliotoxin (9), 6-acetylbis(methylthio)gliotoxin (10), bisdethiobis(methylthio) gliotoxin (11) and bis- $N$-norgliovictin (13), were obtained when grown in glucose-containing medium (GluPY medium). This is the first report of compounds 3, 4, 6, 9, 10 and 12 as naturally occurring. Their structures were determined mainly by MS, 1D and 2D NMR data. The possible biosynthetic pathways of gliotoxin-related analogues and neosartin $\mathrm{C}$ were proposed. The antibacterial activity of compounds $\mathbf{2}-\mathbf{1 4}$ and the cytotoxic activity of compounds 4, 5 and 7-13 were evaluated. Their structure-activity relationships are also preliminarily discussed.

Keywords: marine fungus; Neosartorya pseudofischeri; neosartin; diketopiperazine; antibacterial activity; cytotoxic activity

\section{Introduction}

Marine organism, such as sponges, soft corals and invertebrates, host numerous fungi. These marine fungi are an important source of structurally unique and biologically active natural products. Recent studies revealed that the cryptic biosynthetic pathways of fungi can be activated and the chemical diversity of their metabolites can be maximized by alternating their cultivation parameters systematically, such as the components of the media [1,2], co-culture $[3,4]$, feeding precursors $[5,6]$ and the addition of enzyme inhibitors [7,8]. For example, marine fungus Chondrostereum sp., which was isolated from soft coral Sarcophyton tortuosum, grows in different culture media and can produce various novel bioactive hirsutane-type sesquiterpenoids [9-12].

In recent years, we conducted research on the metabolites of marine fungi isolated from starfish Acanthaster planci and obtained a series of novel and/or bioactive metabolites [13-16]. In the current work, a marine fungus, Neosartorya pseudofischeri, was isolated from the inner tissue of Acanthaster planci. Neosartorya is a sexual state of Aspergillus section Fumigati [17]; however, unlike Aspergillus, the reports on the secondary metabolites of Neosartorya sp. have been relatively rare. The limited literature showed that most of the metabolites from Neosartorya species were cytotoxic nitrogenous-containing compounds [18,19]. In an attempt to explore the biosynthetic potentials, GlyPY (glycerol $10 \mathrm{~g}$, peptone $5 \mathrm{~g}$, yeast extract $2 \mathrm{~g}, \mathrm{CaCO}_{3} 1 \mathrm{~g}$, sea water $1 \mathrm{~L}$ ) and GluPY (glucose $10 \mathrm{~g}$, peptone $5 \mathrm{~g}$, yeast extract $2 \mathrm{~g}$, sea water $1 \mathrm{~L}, \mathrm{pH}$ 7.5) media were separately used to culture the fungus, Neosartorya pseudofischeri. Both of the EtOAc extracts of two different culture conditions showed potent cytotoxicity against cancer cell line HCT-116 with the IC50 values lower than 
$20 \mu \mathrm{g} / \mathrm{mL}$. The HPLC traces of these two EtOAc extracts also displayed distinct components and content differences (Supplementary Figure S1). Purification of the extract of GlyPY medium afforded two novel diketopiperazines, neosartins A and B (1 and 2), together with six biogenetically-related known diketopiperazines, 1,2,3,4-tetrahydro-2,3-dimethyl-1,4-dioxopyrazino[1,2-a]indole (3), 1,2,3,4-tetrahydro-2-methyl-3-methylene-1,4-dioxopyrazino[1,2-a]indole (4), 1,2,3,4-tetrahydro-2-meth yl-1,3,4-trioxopyrazino[1,2-a]indole (5), 6-acetylbis(methylthio) gliotoxin (10), bisdethiobis (methylthio)gliotoxin (11), didehydrobisdethiobis(methylthio)gliotoxin (12) and $\mathrm{N}$-methyl-1H-indo le-2-carboxamide (6). Isolation of the extract of GluPY medium gave a new alkaloid, neosartin C (14), known compounds, $\alpha$-pyrone meroterpenoid pyripyropene A (15), gliotoxin (7) and gliotoxin analogues, acetylgliotoxin (8), reduced gliotoxin (9), 6-acetylbis(methylthio)gliotoxin (10), bisdethiobis(methylthio)gliotoxin (11) and bis- $N$-norgliovictin (13) (Figure 1). In this paper, we report the isolation, structural elucidation, proposed biosynthetic pathways, bioactivities and structure-activity relationships of these compounds.

Figure 1. Structures of compounds 1-15.

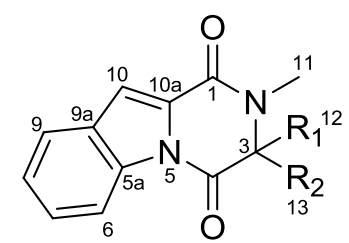

$1 \mathrm{R}_{1}=\mathrm{CH}_{3}, \mathrm{R}_{2}=\mathrm{OCH}_{3}$

$2 \mathrm{R}_{1}=\mathrm{CH}_{2} \mathrm{OH}, \mathrm{R}_{2}=\mathrm{OCH}_{3}$

$3 \mathrm{R}_{1}=\mathrm{CH}_{3}, \mathrm{R}_{2}=\mathrm{H}$<smiles></smiles>

$4 \mathrm{R}=\mathrm{CH}_{2}$

$5 \mathrm{R}=\mathrm{O}$

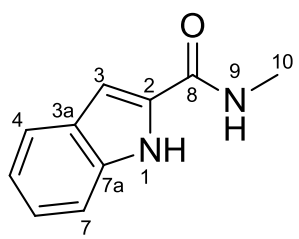

6

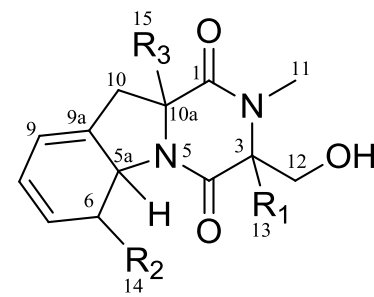

$9 \mathrm{R}_{1}=\mathrm{R}_{3}=\mathrm{SH}, \mathrm{R}_{2}=\mathrm{OH}$

$7 \mathrm{R}=\mathrm{OH}$

$10 \mathrm{R}_{1}=\mathrm{R}_{3}=\mathrm{SCH}_{3}, \mathrm{R}_{2}=\mathrm{OAc}$

$11 \mathrm{R}_{1}=\mathrm{R}_{3}=\mathrm{SCH}_{3}, \mathrm{R}_{2}=\mathrm{OH}$

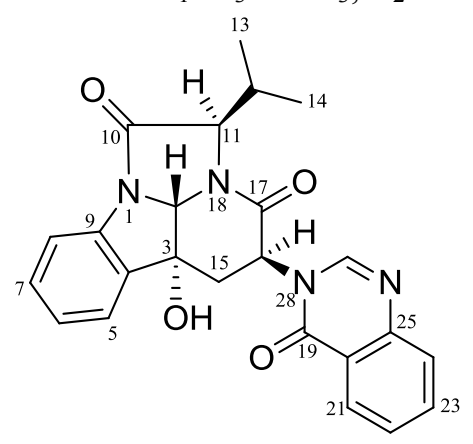

14

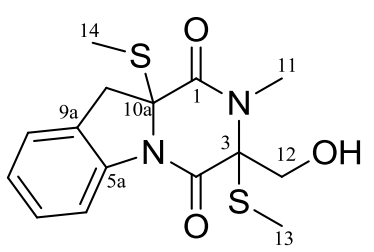

12<smiles>CSC1(CO)NC(=O)C(Cc2ccccc2)(SC)NC1=O</smiles>

13

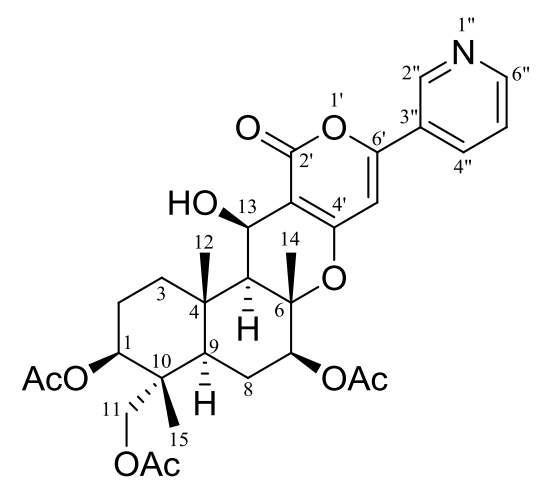

15 


\section{Results and Discussion}

\subsection{Structural Elucidation}

Compound 1 was obtained as a yellowish solid. The molecular formula was determined as $\mathrm{C}_{14} \mathrm{H}_{14} \mathrm{~N}_{2} \mathrm{O}_{3}$ from the HREIMS peak at $\mathrm{m} / z$ 258.0998 [M] ${ }^{+}$(calcd. 258.0999) (Supplementary Figures S2 and S3), implying nine degrees of unsaturation. The IR spectrum indicated the presence of a carbonyl group $\left(1680 \mathrm{~cm}^{-1}\right)$ and a benzene ring $\left(3073,1574\right.$ and $\left.1507 \mathrm{~cm}^{-1}\right)$. UV maxima at 211, 243 and $295 \mathrm{~nm}$ displayed the conjugated system containing a benzene ring. The ${ }^{13} \mathrm{C}$ NMR and DEPT spectra displayed three methyls, five methines and six quaternary carbons (Table 1 and Supplementary Figures S4-S6). Two quaternary carbons at $\delta_{\mathrm{C}} 163.7$ and 156.7 are amide carbonyls. The amide protons were substituted for the lack of the corresponding signals in IR and ${ }^{1} \mathrm{H} \mathrm{NMR}$ spectra in $\mathrm{CDCl}_{3}$. Eight carbon resonance signals appeared in the region of $\delta_{\mathrm{C}} 114.9 \sim 134.8$. Among them, four aromatic methines $\left(\delta_{\mathrm{C}} 116.8, \delta_{\mathrm{H}} 8.50(\mathrm{~d}, 8.0, \mathrm{H}-6) ; \delta_{\mathrm{C}} 125.8, \delta_{\mathrm{H}} 7.43\right.$ (ddd, 8.0, 8.0, 0.8, H-7); $\delta_{\mathrm{C}} 128.2$, $\delta_{\mathrm{H}} 7.55(\mathrm{ddd}, 8.0,8.0,0.8, \mathrm{H}-8)$; and $\delta_{\mathrm{C}} 122.7, \delta_{\mathrm{H}} 7.72(\mathrm{~d}, 8.0, \mathrm{H}-9)$ ) established the partial structure $-\mathrm{CH}-\mathrm{CH}-\mathrm{CH}-\mathrm{CH}-$ based on the ${ }^{1} \mathrm{H}-{ }^{1} \mathrm{H}$ COSY correlations of $\mathrm{H}-6 / \mathrm{H}-7, \mathrm{H}-7 / \mathrm{H}-8$ and H-8/H-9 (Figure 2, Supplementary Figure S7). Further analysis on the HMBC correlations of H-6/C-5a and H-9/C-9a suggested a 1,2-disubstutited benzene ring in the molecule. The methine $\left(\delta_{\mathrm{C}} 114.9\right.$, $\left.\delta_{\mathrm{H}} 7.49, \mathrm{~s}\right)$ and a quaternary carbon $\mathrm{C}-10 \mathrm{a}$ at $\delta_{\mathrm{C}} 127.8$ constructed an additional trisubstituted double bond. The HMBC correlations of H-9/C-10, H-10/C-9a, H-10/C-10a and H-10/C-1 revealed the trisubstituted double bond connected to a benzene ring and an amide carbonyl. Methyl group $\mathrm{C}-11$ at $\delta_{\mathrm{H}} 3.13$ ( $\delta_{\mathrm{C}} 26.6$ ) showed HMBC correlations with amide carbonyl C-1 and quaternary carbon C-3, so it was connected to amide nitrogen N-2. Methyl group C-12 at $\delta_{\mathrm{H}} 1.81\left(\delta_{\mathrm{C}} 25.3\right)$ was connected to C-3 based on the HMBC correlation with the amide carbon C-4 and quaternary carbon C-3. The methoxyl group was also located at C-3, because it showed an HMBC correlation with C-3 (Supplementary Figure S8). Finally, in order to connect the remaining open bonds, C-5a must be linked to the nitrogen atom at the 5-position to form the additional five-membered ring. In the NOESY spectrum, the correlations among the protons of three methyl groups and $\mathrm{H}-9 / \mathrm{H}-10$, confirmed the connection (Supplementary Figure S9). Therefore, compound 1 was established as 1,2,3,4-tetrahydro-3-methoxyl-2, 3-dimethyl-1,4-dioxopyrazino[1,2-a]indole, trivially named neosartin A.

Compound 2 was isolated as a yellowish solid. The molecular formula was established as $\mathrm{C}_{14} \mathrm{H}_{14} \mathrm{~N}_{2} \mathrm{O}_{4}$ based on the HREIMS peak at $m / z$ 274.0947 $[\mathrm{M}]^{+}$and ${ }^{13} \mathrm{C}$ NMR data (Table 1, and Supplementary Figures S11 and S13). The ${ }^{13} \mathrm{C}$ NMR and DEPT spectra displayed two methyls, one methylene, five methines and six quaternary carbons. The NMR data of compound 2 were very similar to those of compound 1 (Figure 2 and Supplementary Figures S12-S17). By comparison of their NMR data, a quick identification was made that the methyl C-12 $\left(\delta_{\mathrm{C}} 25.3, \delta_{\mathrm{H}} 1.81\right.$, s) in $\mathbf{1}$ was replaced by an oxymethylene $\left(\delta_{\mathrm{C}} 64.9, \delta_{\mathrm{H}} 4.18, \mathrm{~d}, J=10.7 \mathrm{~Hz} ; 3.99, \mathrm{~d}, J=10.7 \mathrm{~Hz}\right)$ in $\mathbf{2}$. Therefore, the structure of 2 was elucidated as 1,2,3,4-tetrahydro-3-methoxyl-3-hydroxylmethyl-2-methyl-1,4-dioxopyrazino[1,2-a] indole, commonly named neosartin B.

Compounds 1 and $\mathbf{2}$ did not show optical activity in circular dichroism (CD) spectra; thus, they existed as a racemic mixture of $3 R$ and $3 S$.

Compound 3 was isolated as a white solid. The molecular formula was established as $\mathrm{C}_{13} \mathrm{H}_{12} \mathrm{~N}_{2} \mathrm{O}_{2}$ based on the LREIMS molecular ion at $\mathrm{m} / z 228$ and the NMR data (Table 1 and Supplementary 
Figure S18). Its NMR spectra data closely resembled those of $\mathbf{1}$ except for the methoxy group in $\mathbf{1}$, which was replaced with a hydrogen atom in $\mathbf{3}$, and that correlated with $\mathrm{C}-3$ in the HMBC spectrum. Due to the vicinal coupling with methyl group $\mathrm{C}-12$, the ${ }^{1} \mathrm{H}$ signal at $\delta 4.33$ appeared as a typical quartet with $J=7.2 \mathrm{~Hz}$. The structure of 3 was confirmed by ${ }^{1} \mathrm{H}-{ }^{1} \mathrm{H}$ COSY, HMBC and NOESY data (Supplementary Figures S19-S24). Compound 3 was determined to be 1,2,3,4-tetrahydro-2, 3-dimethyl-1,4-dioxopyrazino[1,2-a]indole. It was once synthesized by heating anhydrodethiogliotoxin with acetic anhydride [20]; however, this is the first time that the detailed NMR data have been presented.

Table 1. ${ }^{1} \mathrm{H}$ and ${ }^{13} \mathrm{C}$ NMR data of compounds $\mathbf{1}-\mathbf{3}$ at $400 / 100 \mathrm{MHz}$, respectively, in $\mathrm{CDCl}_{3}, \delta$ in ppm.

\begin{tabular}{|c|c|c|c|c|c|c|c|c|c|}
\hline \multirow{2}{*}{ Position } & \multicolumn{3}{|r|}{1} & \multicolumn{3}{|r|}{2} & \multicolumn{3}{|r|}{3} \\
\hline & $\delta \mathbf{C}$ & Type & $\delta_{H}$, mult., $(J$ in Hz) & $\delta_{\mathbf{C}}$ & Type & $\delta_{\mathrm{H}}$, mult., $(J$ in $\mathrm{Hz})$ & $\delta_{\mathbf{C}}$ & Type & $\delta_{\mathrm{H}}$, mult., $(J$ in $\mathrm{Hz})$ \\
\hline 1 & 156.7 & $\mathrm{C}$ & & 158.5 & $\mathrm{C}$ & & 156.2 & $\mathrm{C}$ & \\
\hline 2 & & $\mathrm{~N}$ & & & $\mathrm{~N}$ & & & $\mathrm{~N}$ & \\
\hline 3 & 90.7 & $\mathrm{C}$ & & 93.6 & $\mathrm{C}$ & & 60.2 & $\mathrm{CH}$ & $4.33, \mathrm{q}(7.2)$ \\
\hline 4 & 163.7 & $\mathrm{C}$ & & 162.9 & $\mathrm{C}$ & & 165.5 & $\mathrm{C}$ & \\
\hline 5 & & $\mathrm{~N}$ & & & $\mathrm{~N}$ & & & $\mathrm{~N}$ & \\
\hline $5 \mathrm{a}$ & 129.2 & $\mathrm{C}$ & & 128.8 & $\mathrm{C}$ & & 129.2 & $\mathrm{C}$ & \\
\hline 6 & 116.9 & $\mathrm{CH}$ & 8.49, dd $(8.0,0.8)$ & 116.6 & $\mathrm{CH}$ & $8.47, \mathrm{dd}(7.6,0.8)$ & 116.5 & $\mathrm{CH}$ & $8.43, \mathrm{~d}(8.0)$ \\
\hline 7 & 128.2 & $\mathrm{CH}$ & 7.55 , ddd $(8.0,8.0,0.8)$ & 128.1 & $\mathrm{CH}$ & 7.49, ddd $(7.6,7.6,0.8)$ & 127.8 & $\mathrm{CH}$ & $7.51, \mathrm{dd}(8.0,8.0)$ \\
\hline 8 & 125.8 & $\mathrm{CH}$ & 7.43, ddd $(8.0,8.0,0.8)$ & 125.7 & $\mathrm{CH}$ & 7.26 , ddd $(7.6,7.6,0.8)$ & 125.4 & $\mathrm{CH}$ & $7.40, \mathrm{dd}(8.0,8.0)$ \\
\hline 9 & 122.7 & $\mathrm{CH}$ & $7.72, \mathrm{dd}(8.0,0.8)$ & 122.5 & $\mathrm{CH}$ & $7.32, \mathrm{dd}(7.6,0.8)$ & 122.5 & $\mathrm{CH}$ & $7.70, \mathrm{~d}(8.0)$ \\
\hline $9 \mathrm{a}$ & 134.8 & $\mathrm{C}$ & & 134.5 & $\mathrm{C}$ & & 134.8 & $\mathrm{C}$ & \\
\hline 10 & 114.9 & $\mathrm{CH}$ & $7.50, \mathrm{~s}$ & 115.0 & $\mathrm{CH}$ & 7.19, s & 114.1 & $\mathrm{CH}$ & $7.44, \mathrm{~s}$ \\
\hline $10 \mathrm{a}$ & 127.8 & $\mathrm{C}$ & & 127.5 & $\mathrm{C}$ & & 128.5 & $\mathrm{C}$ & \\
\hline 11 & 26.6 & $\mathrm{CH}_{3}$ & $3.13, \mathrm{~s}$ & 26.5 & $\mathrm{CH}_{3}$ & $3.14, \mathrm{~s}$ & 31.8 & $\mathrm{CH}_{3}$ & $3.16, \mathrm{~s}$ \\
\hline 12 & 25.3 & $\mathrm{CH}_{3}$ & $1.81, \mathrm{~s}$ & 64.9 & $\mathrm{CH}_{2}$ & $\begin{array}{l}4.17, \mathrm{~d}(11.6) \\
4.02, \mathrm{~d}(11.6)\end{array}$ & 19.8 & $\mathrm{CH}_{3}$ & $1.71, \mathrm{~d}(7.2)$ \\
\hline 13 & 52.0 & $\mathrm{O}_{\underline{C H}}$ & $3.20, \mathrm{~s}$ & 52.0 & $\mathrm{O} \underline{\mathrm{CH}_{3}}$ & $3.24, \mathrm{~s}$ & & & \\
\hline $12-\mathrm{OH}$ & & & & & & 2.04, brs & & & \\
\hline
\end{tabular}

Compound 4 contains a typical terminal $\mathrm{C}=\mathrm{C}$ double bond $\left(\delta_{\mathrm{C}} 137.7, \mathrm{C}-3 ; \delta_{\mathrm{C}} 106.0, \delta_{\mathrm{H}} 6.15\right.$, s; $5.25, \mathrm{~s}, \mathrm{C}-12)$. Its structure was elucidated as 1,2,3,4-tetrahydro-2-methyl-3-methylene-1,4-dio xopyrazino[1,2-a]indole by analysis of its spectral data (Table 2, Figure 2 and Supplementary Figures S25-S31). Compound 4 was previously obtained as the conversion product of gliotoxin (7) by passing through a column of alkaline alumina at $20{ }^{\circ} \mathrm{C}$ [21]. Compound 5 was deduced as 1,2,3,4-tetrahydro-2-me thyl-1,3,4-trioxopyrazino[1,2-a]indole by careful analysis of the MS and NMR data (Table 2, Figure 2 and Supplementary Figures S32-S38). Compound 5 was firstly reported in 1945 as a degradation product of gliotoxin by heating with selenium [22]. It was also isolated from the culture of Penicillium terlikowskii [23]. Compound $\mathbf{6}$ was obtained as a white solid. The molecular formula was established as $\mathrm{C}_{10} \mathrm{H}_{10} \mathrm{~N}_{2} \mathrm{O}$ on the basis of HREIMS (m/z 174.0789 [M] $]^{+}$, calcd. 174.0788) and NMR data (Table 2 and Supplementary Figures S39-S45). The structure of 6 was elucidated as $\mathrm{N}$-methyl-1H-indole-2-carboxamide by analysis on the 1D and 2D NMR (HMQC, HMBC and ${ }^{1} \mathrm{H}-{ }^{1} \mathrm{H}$ COSY) (Table 2 and Figure 2). It is unprecedented that compound 6 was obtained from a natural source. 
Figure 2. ${ }^{1} \mathrm{H}-{ }^{1} \mathrm{H}$ COSY (bold line) and the main HMBC (arrows) correlations of compounds $\mathbf{1}-\mathbf{6}$.

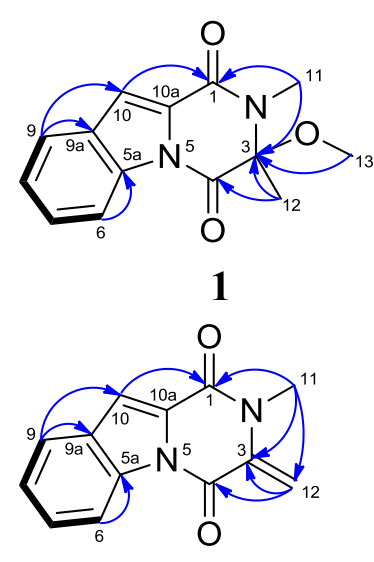

4

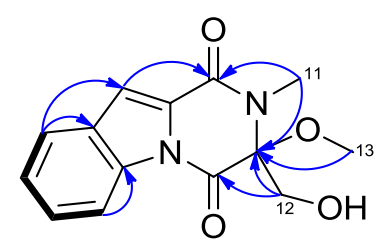

2

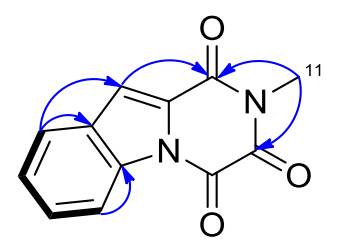

5
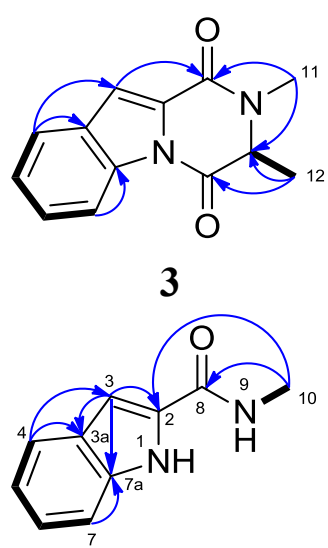

6

Table 2. ${ }^{1} \mathrm{H}$ and ${ }^{13} \mathrm{C}$ NMR data of compounds 4-6 at 400/100 MHz, respectively, $\delta$ in ppm.

\begin{tabular}{|c|c|c|c|c|c|c|c|c|c|c|}
\hline \multirow{2}{*}{ Position } & \multicolumn{3}{|r|}{$4^{a}$} & \multicolumn{3}{|r|}{$5^{b}$} & \multirow{2}{*}{ Position } & \multicolumn{3}{|r|}{$6^{\mathrm{c}}$} \\
\hline & $\boldsymbol{\delta}_{\mathbf{C}}$ & Type & $\delta_{H}$, mult., $(J$ in Hz$)$ & $\delta_{\mathbf{C}}$ & Type & $\delta_{\mathrm{H}}$, mult., $(J$ in $\mathrm{Hz})$ & & $\delta_{\mathbf{C}}$ & Type & $\delta_{\mathrm{H}}$, mult., $(J$ in $\mathrm{Hz})$ \\
\hline 1 & 154.4 & $\mathrm{C}$ & & 157.0 & $\mathrm{C}$ & & 1 & & NH & 11.04, brs \\
\hline 2 & & $\mathrm{~N}$ & & & $\mathrm{~N}$ & & 2 & 132.8 & $\mathrm{C}$ & \\
\hline 3 & 137.7 & $\mathrm{C}$ & & 156.8 & $\mathrm{C}$ & & 3 & 102.7 & $\mathrm{CH}$ & $7.07, \mathrm{~s}$ \\
\hline 4 & 154.9 & $\mathrm{C}$ & & 149.9 & $\mathrm{C}$ & & $3 a$ & 137.8 & $\mathrm{C}$ & \\
\hline 5 & & $\mathrm{~N}$ & & & $\mathrm{~N}$ & & 4 & 122.5 & $\mathrm{CH}$ & $7.61, \mathrm{~d}(8.0)$ \\
\hline $5 \mathrm{a}$ & 128.9 & $\mathrm{C}$ & & 128.3 & $\mathrm{C}$ & & 5 & 124.5 & $\mathrm{CH}$ & $7.22, \mathrm{dd}(8.0,8.0)$ \\
\hline 6 & 117.0 & $\mathrm{CH}$ & $8.50, \mathrm{~d}(8.0)$ & 115.8 & $\mathrm{CH}$ & $8.32, \mathrm{~d}(8.0)$ & 6 & 120.9 & $\mathrm{CH}$ & $7.06, \mathrm{dd}(8.0,8.0)$ \\
\hline 7 & 128.2 & $\mathrm{CH}$ & $7.53, \mathrm{dd}(8.0,8.0)$ & 129.0 & $\mathrm{CH}$ & $7.63, \mathrm{~d}(8.0,8.0)$ & 7 & 113.2 & $\mathrm{CH}$ & $7.58, \mathrm{~d}(8.0)$ \\
\hline 8 & 125.5 & $\mathrm{CH}$ & 7.40, dd $(8.0,8.0)$ & 125.7 & $\mathrm{CH}$ & $7.47, \mathrm{~d}(8.0,8.0)$ & $7 \mathrm{a}$ & 128.9 & $\mathrm{C}$ & \\
\hline 9 & 122.8 & $\mathrm{CH}$ & $7.72, \mathrm{~d}(8.0)$ & 123.9 & $\mathrm{CH}$ & $7.88, \mathrm{~d}(8.0)$ & 8 & 163.0 & $\mathrm{C}$ & \\
\hline $9 a$ & 135.6 & $\mathrm{C}$ & & 135.4 & $\mathrm{C}$ & & 9 & & $\mathrm{NH}$ & 7.83 , brs \\
\hline 10 & 115.3 & $\mathrm{CH}$ & $7.51, \mathrm{~s}$ & 116.3 & $\mathrm{CH}$ & $7.72, \mathrm{~s}$ & 10 & 26.4 & $\mathrm{CH}_{3}$ & $2.97, \mathrm{~s}$ \\
\hline $10 \mathrm{a}$ & 127.6 & $\mathrm{C}$ & & 127.7 & $\mathrm{C}$ & & & & & \\
\hline 11 & 29.6 & $\mathrm{CH}_{3}$ & $3.41, \mathrm{~s}$ & 26.6 & $\mathrm{CH}_{3}$ & $3.22, \mathrm{~s}$ & & & & \\
\hline 12 & 106.0 & $\mathrm{CH}_{2}$ & $6.15, \mathrm{~s} ; 5.25, \mathrm{~s}$ & & & & & & & \\
\hline
\end{tabular}

${ }^{\mathrm{a}}$ Measured in $\mathrm{CDCl}_{3} .{ }^{\mathrm{b}}$ Measured in DMSO- $d_{6} \cdot{ }^{\mathrm{c}}$ Measured in acetone- $d_{6}$.

Compound 7 was identified as gliotoxin by comparing the data with the literature values [24,25] (Supplementary Figures S46-S48). The NMR spectra data of compound $\mathbf{8}$ closely resembled those of 7, except for one additional ${ }^{1} \mathrm{H}$ resonance signal of the acetyl group. Its structure was identified as acetylgliotoxin, which was isolated from fungus strain FO2047 previously, and showed broad activities, including inhibition of fungi, bacteria and viruses [26] (Table 3 and Supplementary Figures S49 and S50). Compound 9, having NMR data similar to those of 7, was identified as reduced gliotoxin, which was the reduced dithiol form of 7 (Table 3 and Supplementary Figures S51 and S52). Daniel et al. found that gliotoxin (7) was generated from the corresponding dithiol (9) by a novel FAD-dependent dithiol oxidase, GliT [27]. Although 4-6, 8 and 9 are known compounds, their detailed NMR data were never reported previously. 
Table 3. ${ }^{1} \mathrm{H}$ and ${ }^{13} \mathrm{C}$ NMR data of compounds 8 and 9 in $\mathrm{CDCl}_{3}, \delta$ in ppm.

\begin{tabular}{|c|c|c|c|c|c|c|}
\hline \multirow{2}{*}{ Position } & \multicolumn{3}{|r|}{$8^{\mathrm{a}}$} & \multicolumn{3}{|r|}{$9^{b}$} \\
\hline & $\delta \mathrm{C}$ & Type & $\delta_{\mathrm{H}}$, mult., $(J$ in $\mathrm{Hz})$ & $\delta \mathrm{C}$ & Type & $\delta_{\mathrm{H}}$, mult., $(J$ in $\mathrm{Hz})$ \\
\hline 1 & 168.4 & $\mathrm{C}$ & & 169.7 & $\mathrm{C}$ & \\
\hline 2 & & $\mathrm{~N}$ & & & $\mathrm{~N}$ & \\
\hline 3 & 78.2 & $\mathrm{C}$ & & 78.6 & $\mathrm{C}$ & \\
\hline 4 & 165.9 & $\mathrm{C}$ & & 168.1 & $\mathrm{C}$ & \\
\hline 5 & & $\mathrm{~N}$ & & & $\mathrm{~N}$ & \\
\hline $5 a$ & 64.9 & $\mathrm{CH}$ & $5.36, \mathrm{~d}(14.1)$ & 70.6 & $\mathrm{CH}$ & $4.77, \mathrm{~d}(13.2)$ \\
\hline 6 & 74.3 & $\mathrm{CH}$ & $5.82, \mathrm{~d}(14.1)$ & 72.7 & $\mathrm{CH}$ & $5.04, \mathrm{~d}(13.2)$ \\
\hline 7 & 128.6 & $\mathrm{CH}$ & $5.93, \mathrm{~m}$ & 129.8 & $\mathrm{CH}$ & $5.93, \mathrm{~m}$ \\
\hline 8 & 124.9 & $\mathrm{CH}$ & $5.93, \mathrm{~m}$ & 123.1 & $\mathrm{CH}$ & $5.88, \mathrm{~m}$ \\
\hline 9 & 120.7 & $\mathrm{CH}$ & $5.60, \mathrm{~d}(7.5)$ & 120.7 & $\mathrm{CH}$ & $5.75, \mathrm{~m}$ \\
\hline $9 \mathrm{a}$ & 131.7 & $\mathrm{C}$ & & 130.0 & $\mathrm{C}$ & \\
\hline 10 & 41.4 & $\mathrm{CH}_{2}$ & $\begin{array}{l}3.25, \mathrm{~d}(15.0) \\
3.08, \mathrm{~d}(15.0)\end{array}$ & & $\mathrm{CH}_{2}$ & $\begin{array}{l}3.28, \mathrm{~d}(16.2) \\
3.05, \mathrm{~d}(16.2)\end{array}$ \\
\hline $10 \mathrm{a}$ & 77.6 & $\mathrm{C}$ & & 77.2 & $\mathrm{C}$ & \\
\hline 11 & 29.0 & $\mathrm{CH}_{3}$ & $3.10, \mathrm{~s}$ & 28.9 & $\mathrm{CH}_{3}$ & $3.15, \mathrm{~s}$ \\
\hline 12 & 62.4 & $\mathrm{CH}_{2}$ & $\begin{array}{l}4.32, \mathrm{~d}(12.3) \\
4.00, \mathrm{~d}(12.3)\end{array}$ & 62.3 & $\mathrm{CH}_{2}$ & $\begin{array}{l}4.39, \mathrm{~d}(12.0) \\
4.06, \mathrm{~d}(12.0)\end{array}$ \\
\hline 13 & $\begin{array}{c}170.1 \\
21.4 \\
\end{array}$ & $\begin{array}{l}\frac{\mathrm{COCH}_{3}}{\mathrm{CO} \mathrm{CH}_{3}} \\
\end{array}$ & $2.17, \mathrm{~s}$ & & & \\
\hline
\end{tabular}

${ }^{a}{ }^{1} \mathrm{H}$ and ${ }^{13} \mathrm{C}$ NMR data were measured at $300 / 75 \mathrm{MHz} ;{ }^{\mathrm{b}}{ }^{1} \mathrm{H}$ and ${ }^{13} \mathrm{C}$ NMR data were measured at 400/100 $\mathrm{MHz}$. $\mathrm{CDCl}_{3}$.

Compounds 10-13 were identified as 6-acetylbis(methylthio)gliotoxin (10) [28], bisdethiobis (methylthio)gliotoxin (11) [29], didehydrobisdethiobis(methylthio)gliotoxin (12) [30] and bis- $N$-norgliovictin (13) [31], respectively, by comparing their spectroscopic data (Table 4 and Supplementary Figures S53-S60) with the literature values. In the literature, only the ${ }^{1} \mathrm{H}$ NMR data of compound 12 were reported; here, we report the detailed ${ }^{1} \mathrm{H}$ and ${ }^{13} \mathrm{C}$ NMR data. The ${ }^{13} \mathrm{C}$ NMR data of compound 13 recorded in DMSO- $d_{6}$ were shifted about 0.7 2.5 ppm to a higher field compared to the data reported for pyridine- $d 5$.

Compound 14 was obtained as a yellow solid. The molecular formula was deduced as $\mathrm{C}_{24} \mathrm{H}_{22} \mathrm{~N}_{4} \mathrm{O}_{4}$ from the HREIMS peak at $\mathrm{m} / z$ 430.1635 [M] ${ }^{+}$(calcd. 430.1636), implying 16 degrees of unsaturation (Supplementary Figure S62). The ${ }^{13} \mathrm{C}$ NMR and DEPT spectra displayed twenty-four carbons, which were classified into two methyls, one methylene, thirteen methines and eight quaternary carbons (Table 5, Supplementary Figures S63 and S64). The chemical shifts of sixteen carbons were located at $\delta_{\mathrm{C}}$ 115.0-172.0, corresponding to the aromatic or double-bond carbons. The ${ }^{1} \mathrm{H}$ NMR spectrum showed eight proton signals in the downfield region $\left(\delta_{\mathrm{H}} 7.15 \sim 8.20\right)$ with the coupling constants being about 7.6, suggesting at least two phenyl groups in the molecule. By analysis of the HMQC spectrum, the ${ }^{1} \mathrm{H}$ and ${ }^{13} \mathrm{C}$ NMR data of each carbon were definitely assigned. The ${ }^{1} \mathrm{H}-{ }^{1} \mathrm{H}$ COSY correlations of $\mathrm{H}-15 / \mathrm{H}-16, \mathrm{H}-11 / \mathrm{H}-12, \mathrm{H}-12 / \mathrm{H}-13$ and $\mathrm{H}-12 / \mathrm{H}-14$ deduced the presence of two partial structures, $-\mathrm{CH}_{2} \mathrm{CH}$ - and $-\mathrm{CHCH}\left(\mathrm{CH}_{3}\right)_{2}$, respectively (Figure 3a, Supplementary Figure S66). Furthermore, the COSY correlations of H-5/H-6, H-6/H-7, H-7/H-8, H-21/H-22, H-22/H-23 and H-23/H-24 indicate that there are two - $\mathrm{CHCHCHCH}$ - in the molecule. HMBC correlations of H-5/C-4, H-6/C-4, H-7/C-9, 
H-8/C-9, H-21/C-20, H-21/C-25, H-22/C-20, H-23/C-25 and H-24/C-25 further confirmed the presence of two disubstituted benzene rings. Three quaternary carbons at $\delta_{\mathrm{c}} 171.4(\mathrm{C}-10), 163.6$ (C-17) and 161.7 (C-19) are amide carbonyl groups. HMBC correlations of H-11/C-10, H-16/C-17, $\mathrm{H}-21 / \mathrm{C}-19$ revealed that three amide carbonyl groups were connected to $-\mathrm{CHCH}\left(\mathrm{CH}_{3}\right)_{2},-\mathrm{CH}_{2} \mathrm{CH}-$ and a disubstituted benzene ring, respectively. The hydroxyl group at $\delta_{\mathrm{H}} 4.09$ (s) was attached to $\mathrm{C}-3$ ( $\left.\delta_{\mathrm{C}} 74.8\right)$, and $\mathrm{C}-3$ was connected to the methine $\mathrm{C}-2\left(\delta_{\mathrm{C}} 84.8\right)$ and $\mathrm{C}-15\left(\delta_{\mathrm{C}} 36.5\right)$ based on the HMBC correlations of H-2/C-3 and H-15/C-3. The planar structure of 14 was finally established by the HMBC correlations of H-2/C-10, H-5/C-3, H-15/C-3, H-16/C-19, H-16/C-27 and H-27/C-25 (Supplementary Figure S67).

Table 4. ${ }^{1} \mathrm{H}$ and ${ }^{13} \mathrm{C}$ NMR data of compounds 12 and $13, \delta$ in ppm.

\begin{tabular}{|c|c|c|c|c|c|c|c|}
\hline \multirow{2}{*}{ Position } & \multicolumn{3}{|r|}{$12^{a}$} & \multirow{2}{*}{ Position } & \multicolumn{3}{|r|}{$13^{b}$} \\
\hline & $\boldsymbol{\delta}_{\mathbf{C}}$ & Type & $\delta_{\mathrm{H}}$, mult., $(J$ in $\mathrm{Hz})$ & & $\delta_{\mathbf{C}}$ & Type & $\delta_{\mathrm{H}}$, mult., $(J$ in $\mathrm{Hz})$ \\
\hline 1 & 166.0 & $\mathrm{C}$ & & 1 & 165.2 & $\mathrm{C}$ & \\
\hline 2 & & $\mathrm{~N}$ & & 2 & & $\mathrm{NH}$ & 8.95, brs \\
\hline 3 & 71.7 & $\mathrm{C}$ & & 3 & 65.8 & $\mathrm{C}$ & \\
\hline 4 & 161.8 & $\mathrm{C}$ & & 4 & 165.0 & $\mathrm{C}$ & \\
\hline 5 & & $\mathrm{~N}$ & & 5 & & $\mathrm{NH}$ & 8.40, brs \\
\hline $5 a$ & 128.9 & $\mathrm{C}$ & & 6 & 65.6 & $\mathrm{C}$ & \\
\hline 6 & 127.9 & $\mathrm{CH}$ & $8.03, \mathrm{~d}(8.0)$ & 7 & 43.3 & $\mathrm{CH}_{2}$ & $3.33, \mathrm{~d}(6.0) ; 3.31, \mathrm{~d}(6.0)$ \\
\hline 7 & 126.2 & $\mathrm{CH}$ & $7.31, \mathrm{dd}(8.0,8.0)$ & 8 & 135.0 & $\mathrm{C}$ & \\
\hline 8 & 125.2 & $\mathrm{CH}$ & $7.19, \mathrm{dd}(8.0,8.0)$ & $9 / 9^{\prime}$ & 130.0 & $\mathrm{CH}$ & $7.20, \mathrm{~m}$ \\
\hline 9 & 118.1 & $\mathrm{CH}$ & $7.30, \mathrm{~d}(8.0)$ & $10 / 10^{\prime}$ & 127.6 & $\mathrm{CH}$ & $7.20, \mathrm{~m}$ \\
\hline $9 a$ & 140.6 & $\mathrm{C}$ & & 11 & 126.5 & $\mathrm{CH}$ & $7.20, \mathrm{~m}$ \\
\hline 10 & 39.6 & $\mathrm{CH}_{2}$ & $4.50, \mathrm{~d}(12.0) ; 3.96, \mathrm{~d}(12.0)$ & 12 & 64.8 & $\mathrm{CH}_{2}$ & $3.52, \mathrm{~d}(18.0) ; 3.00, \mathrm{~d}(18.0)$ \\
\hline $10 \mathrm{a}$ & 70.8 & $\mathrm{C}$ & & 13 & 12.8 & $\mathrm{CH}_{3}$ & $2.11, \mathrm{~s}$ \\
\hline 11 & 28.9 & $\mathrm{CH}_{3}$ & $3.20, \mathrm{~s}$ & 14 & 13.5 & $\mathrm{CH}_{3}$ & $2.29, \mathrm{~s}$ \\
\hline 12 & 63.9 & $\mathrm{CH}_{2}$ & $3.62, \mathrm{~d}(16.8) ; 3.51, \mathrm{~d}(16.8)$ & & & & \\
\hline 13 & 14.5 & $\mathrm{CH}_{3}$ & $2.32, \mathrm{~s}$ & & & & \\
\hline 14 & 13.8 & $\mathrm{CH}_{3}$ & $2.24, \mathrm{~s}$ & & & & \\
\hline
\end{tabular}

${ }^{\text {a }}{ }^{1} \mathrm{H}$ and ${ }^{13} \mathrm{C}$ NMR data were measured at $400 / 100 \mathrm{MHz}$ in $\mathrm{CDCl}_{3} ;{ }^{\mathrm{b}}{ }^{1} \mathrm{H}$ and ${ }^{13} \mathrm{C}$ NMR data were measured at $300 / 75 \mathrm{MHz}$ in DMSO- $d_{6}$.

The relative stereochemistry of $\mathbf{1 4}$ was determined on the basis of NOESY data. The NOESY correlations of $\mathrm{H}-2$ with $\mathrm{H}-12, \mathrm{H}-13$ and $\mathrm{H}-14$ and of $\mathrm{OH}-3$ with $\mathrm{H}-11$ suggested that $\mathrm{H}-2$ and $\mathrm{H}-11$ were placed on the opposite face of the ring system and $\mathrm{H}-11$ and the hydroxyl group at $\mathrm{C}-3$ position were placed on the same face (Figure 3b). No NOESY correlation between H-2 and H-16 was observed (Supplementary Figure S68). Therefore, H-2 was assigned a $\beta$-orientation, whereas OH-3, $\mathrm{H}-11$ and $\mathrm{H}-16$ were determined to have an $\alpha$-orientation.

The NMR data of compound 15 recorded in $\mathrm{CDCl}_{3}$ and acetone- $d_{6}$ showed minor differences (Table 6 and Supplementary Figures S69-S75). By comparison of its spectral data with those reported in the literature, compound 15 was elucidated as pyripyropene A [31]. Pyripyropene A (15), previously isolated from Aspergillus fumigatus FO-1289, showed very potent inhibition of cholesterol acyltransferase (ACAT). 
Table 5. ${ }^{1} \mathrm{H}$ and ${ }^{13} \mathrm{C}$ NMR data of compound 14 at $400 / 100 \mathrm{MHz}$, respectively, in $\mathrm{CDCl}_{3}$, $\delta$ in ppm.

\begin{tabular}{|c|c|c|c|}
\hline Position & $\boldsymbol{\delta}_{\mathrm{C}}$ & Type & $\delta_{\mathrm{H}}$, mult., $(J$ in $\mathrm{Hz})$ \\
\hline 1 & & $\mathrm{~N}$ & \\
\hline 2 & 84.8 & $\mathrm{CH}$ & $5.95, \mathrm{~s}$ \\
\hline 3 & 74.8 & $\mathrm{C}$ & \\
\hline 4 & 135.2 & $\mathrm{C}$ & \\
\hline 5 & 124.7 & $\mathrm{CH}$ & 7.43, brd $(7.6)$ \\
\hline 6 & 126.4 & $\mathrm{CH}$ & $7.15, \mathrm{dd}(7.6,7.6)$ \\
\hline 7 & 130.9 & $\mathrm{CH}$ & $7.35, \mathrm{dd}(7.6,7.6)$ \\
\hline 8 & 115.6 & $\mathrm{CH}$ & $7.53, \mathrm{~d}(7.6)$ \\
\hline 9 & 138.5 & $\mathrm{C}$ & \\
\hline 10 & 171.4 & $\mathrm{C}$ & \\
\hline 11 & 70.3 & $\mathrm{CH}$ & $4.43, \mathrm{~d}(8.4)$ \\
\hline 12 & 30.1 & $\mathrm{CH}$ & $2.35, \mathrm{dqq}(8.4,6.4,6.4)$ \\
\hline 13 & 20.1 & $\mathrm{CH}_{3}$ & $1.17, \mathrm{~d}(6.4)$ \\
\hline 14 & 19.1 & $\mathrm{CH}_{3}$ & $1.21, \mathrm{~d}(6.4)$ \\
\hline 15 & 36.5 & $\mathrm{CH}_{2}$ & $2.49, \mathrm{dd}(15.2,5.2) ; 3.23, \mathrm{dd}(15.2,4.8)$ \\
\hline 16 & 56.8 & $\mathrm{CH}$ & $5.11, \mathrm{dd}(5.2,4.8)$ \\
\hline 17 & 163.6 & $\mathrm{C}$ & \\
\hline 18 & & $\mathrm{~N}$ & \\
\hline 19 & 161.7 & $\mathrm{C}$ & \\
\hline 20 & 121.4 & $\mathrm{C}$ & \\
\hline 21 & 127.4 & $\mathrm{CH}$ & $8.20, \mathrm{dd}(7.6,0.8)$ \\
\hline 22 & 127.8 & $\mathrm{CH}$ & $7.50, \mathrm{dd}(7.6,7.6)$ \\
\hline 23 & 134.9 & $\mathrm{CH}$ & 7.71, ddd $(7.6,7.6,0.8)$ \\
\hline 24 & 126.1 & $\mathrm{CH}$ & $7.64, \mathrm{~d}(7.6)$ \\
\hline 25 & 144.4 & $\mathrm{C}$ & \\
\hline 26 & & $\mathrm{~N}$ & \\
\hline 27 & 147.5 & $\mathrm{CH}$ & $8.61, \mathrm{~s}$ \\
\hline 28 & & $\mathrm{~N}$ & \\
\hline $3-\mathrm{OH}$ & & & 4.09, brs \\
\hline
\end{tabular}

Figure 3. (a) ${ }^{1} \mathrm{H}-{ }^{1} \mathrm{H}$ COSY (bold line), the main HMBC (arrows); and (b) key NOESY correlations of compound 14.

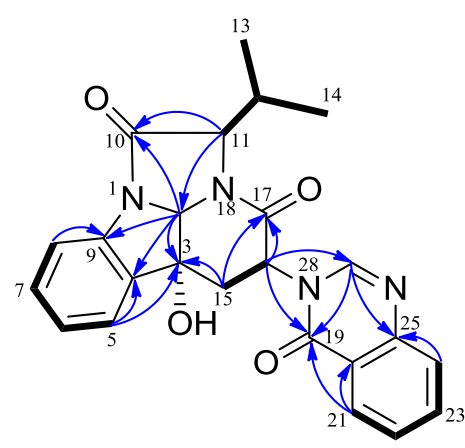

(a)

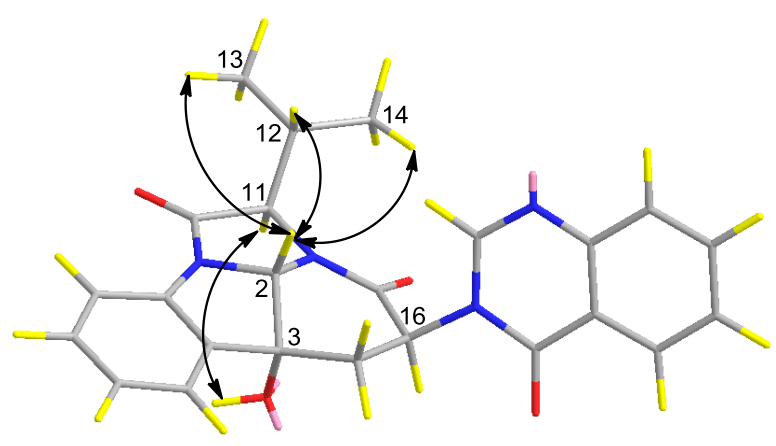

(b) 
Table 6. ${ }^{1} \mathrm{H}$ and ${ }^{13} \mathrm{C}$ NMR data of compound 15 at $600 / 150 \mathrm{MHz}$, respectively, $\delta$ in ppm.

\begin{tabular}{|c|c|c|c|c|c|}
\hline \multirow{2}{*}{ Position } & \multicolumn{3}{|r|}{ In $\mathrm{CDCl}_{3}$} & \multicolumn{2}{|r|}{ In Acetone- $d_{6}$} \\
\hline & $\boldsymbol{\delta}_{\mathbf{C}}$ & Type & $\delta_{\mathrm{H}}$, mult., $(J$ in $\mathrm{Hz})$ & $\boldsymbol{\delta}_{\mathrm{C}}$ & $\delta_{\mathrm{H}}$, mult., $(J$ in $\mathrm{Hz})$ \\
\hline 1 & 73.5 & $\mathrm{CH}$ & $5.00, \mathrm{dd}(10.8,4.8)$ & 74.5 & 4.99, dd $(10.8,4.8)$ \\
\hline 2 & 22.7 & $\mathrm{CH}_{2}$ & $1.83, \mathrm{~m} ; 1.90, \mathrm{~m}$ & 23.7 & $1.85, \mathrm{~m} ; 1.89, \mathrm{~m}$ \\
\hline 3 & 36.2 & $\mathrm{CH}_{2}$ & $1.37, \mathrm{~m} ; 2.16, \mathrm{~m}$ & 36.8 & $1.49, \mathrm{~m} ; 2.17, \mathrm{ddd}(12.6,4.8,4.8)$ \\
\hline 4 & 37.9 & $\mathrm{C}$ & & 38.8 & \\
\hline 5 & 54.7 & $\mathrm{CH}$ & $1.53, \mathrm{~d}(4.2)$ & 55.1 & $1.67, \mathrm{~d}(4.8)$ \\
\hline 6 & 83.3 & $\mathrm{C}$ & & 83.9 & \\
\hline 7 & 77.7 & $\mathrm{CH}$ & $4.78, \mathrm{dd}(12.0,4.8)$ & 79.0 & $4.81, \mathrm{dd}(11.4,4.8)$ \\
\hline 8 & 25.2 & $\mathrm{CH}_{2}$ & $\begin{array}{l}\text { 1.63, ddd }(12.0,12.0,11.4) ; \\
1.78, \operatorname{dd}(11.4,4.8)\end{array}$ & 26.0 & $1.74, \mathrm{~m} ; 1.83, \mathrm{ddd}(10.8,5.4,5.4)$ \\
\hline 9 & 45.4 & $\mathrm{CH}$ & $1.58, \mathrm{~d}(12.0)$ & 46.2 & $1.72, \mathrm{~d}(12.0)$ \\
\hline 10 & 40.3 & $\mathrm{C}$ & & 41.3 & \\
\hline 11 & 64.8 & $\mathrm{CH}_{2}$ & 3.77, d (11.4); 3.70, d (11.4) & 65.6 & $3.76, \mathrm{~d}(12.0) ; 3.72, \mathrm{~d}(12.0)$ \\
\hline 12 & 17.4 & $\mathrm{CH}_{3}$ & $1.43, \mathrm{~s}$ & 17.9 & $1.53, \mathrm{~s}$ \\
\hline 13 & 60.1 & $\mathrm{CH}$ & $4.99, \mathrm{~d}(4.2)$ & 60.5 & $5.00, \mathrm{~d}(4.8)$ \\
\hline 14 & 16.2 & $\mathrm{CH}_{3}$ & $1.69, \mathrm{~s}$ & 16.8 & $1.76, \mathrm{~s}$ \\
\hline 15 & 13.2 & $\mathrm{CH}_{3}$ & $0.83, \mathrm{~s}$ & 13.6 & $0.93, \mathrm{~s}$ \\
\hline $2^{\prime}$ & 163.6 & $\mathrm{C}$ & & 163.3 & \\
\hline $3^{\prime}$ & 103.3 & $\mathrm{C}$ & & 104.2 & \\
\hline $4^{\prime}$ & 162.0 & $\mathrm{C}$ & & 162.7 & \\
\hline $5^{\prime}$ & 99.9 & $\mathrm{CH}$ & $6.48, \mathrm{~s}$ & 100.1 & $6.71, \mathrm{~s}$ \\
\hline $6^{\prime}$ & 156.4 & $\mathrm{C}$ & & 158.1 & \\
\hline $2^{\prime \prime}$ & 145.4 & $\mathrm{CH}$ & $9.05, \mathrm{~s}$ & 147.7 & $9.08, \mathrm{~s}$ \\
\hline $3 "$ & 127.9 & $\mathrm{C}$ & & 128.5 & \\
\hline $4^{\prime \prime}$ & 134.3 & $\mathrm{CH}$ & $8.20, \mathrm{~d}(8.4)$ & 133.8 & 8.24, ddd $(7.8,1.8,1.8)$ \\
\hline $5^{\prime \prime}$ & 124.2 & $\mathrm{CH}$ & 7.51$, brd ( 8.4$)$ & 124.7 & $7.53, \mathrm{dd}(7.8,4.8)$ \\
\hline $6^{\prime \prime}$ & 149.8 & $\mathrm{CH}$ & $8.72, \mathrm{~s}$ & 152.2 & $8.69, \mathrm{~d}(4.8)$ \\
\hline $1-\mathrm{O}-\mathrm{CO}-\mathrm{CH}_{3}$ & 170.5 & $\mathrm{C}$ & & 170.6 & \\
\hline $7-\mathrm{O}-\mathrm{CO}-\mathrm{CH}_{3}$ & 170.0 & $\mathrm{C}$ & & 170.3 & \\
\hline $11-\mathrm{O}-\mathrm{CO}-\mathrm{CH}_{3}$ & 170.9 & $\mathrm{C}$ & & 170.8 & \\
\hline $1-\mathrm{O}-\mathrm{CO}-\mathrm{CH}_{3}$ & 21.1 & $\mathrm{CH}_{3}$ & $2.07, \mathrm{~s}$ & 21.1 & $2.02, \mathrm{~s}$ \\
\hline $7-\mathrm{O}-\mathrm{CO}-\mathrm{CH}_{3}$ & 21.2 & $\mathrm{CH}_{3}$ & $2.15, \mathrm{~s}$ & 21.2 & $2.10, \mathrm{~s}$ \\
\hline $11-\mathrm{O}-\mathrm{CO}-\mathrm{CH}_{3}$ & 20.8 & $\mathrm{CH}_{3}$ & $2.03, \mathrm{~s}$ & 20.7 & $2.00, \mathrm{~s}$ \\
\hline $13-\mathrm{OH}$ & & $\mathrm{OH}$ & 3.06, brs & & 2.90, brs \\
\hline
\end{tabular}

\subsection{Proposed Biosynthetic Pathway}

Diketopiperazines (DKPs) are typically synthesized via nonribosomal peptide synthetases (NRPS) to incorporate more than one amino acid from fungi [32]. Gliotoxin and its analogues have the diketopiperazine core with a disulfide bridge in an oxidized or reduced form. In the biosynthesis of gliotoxin, two-modular nonribosomal peptide synthetase, GliP, incorporates L-phe and L-ser to form dipeptidyl L-phe-L-ser, and under the action of the enzymes, the latter is converted to the corresponding diketopiperazines [33]. Therefore, the biosynthetic pathways of compounds 1-13 were proposed in Figure 4. Catalyzed by GliP, the intermolecular condensation between phenylalanine and serine 
generates an L-phe-L-ser, followed by successive oxidation, sulfuration and epoxidation reactions to obtain the intermediates, $\mathbf{a}-\mathbf{d}$. Then, $\mathbf{a}$ and $\mathbf{b}$ undergo epoxidation, amidation, intramolecular nucleophilic cyclization and $N$-methylation reactions to produce the intermediates, $\mathbf{f}$ and $\mathbf{g}$, respectively. Dehydration of $\mathbf{f}$ furnishes compound $\mathbf{4}$. Compound $\mathbf{4}$ is further hydrogenated and oxidized to afford 3 and $\mathbf{5}$, respectively. The product, $\mathbf{g}$, is sequentially dehydrated, $O$-methylated to form $\mathbf{2}$, followed by the deoxygenation to produce $\mathbf{1}$. After successive amidation and methylation of the thiol groups, the intermediate c generates compound 13. Amidation, intramolecular nucleophilic cyclization and $\mathrm{N}$-methylation of $\mathbf{d}$ afford compound $\mathbf{9}$. Compound $\mathbf{9}$ is $S$-methylated to generate $\mathbf{1 1}$ or dehydrogenated to produce 7, which further undergoes esterification to form $\mathbf{1 0}$ and $\mathbf{8}$, separately. Compound $\mathbf{1 2}$ is produced by the dehydration of $\mathbf{1 1}$, whereas compound $\mathbf{6}$ is the degradation product of 5 .

Figure 4. Proposed biosynthetic pathways of compounds 1-13.

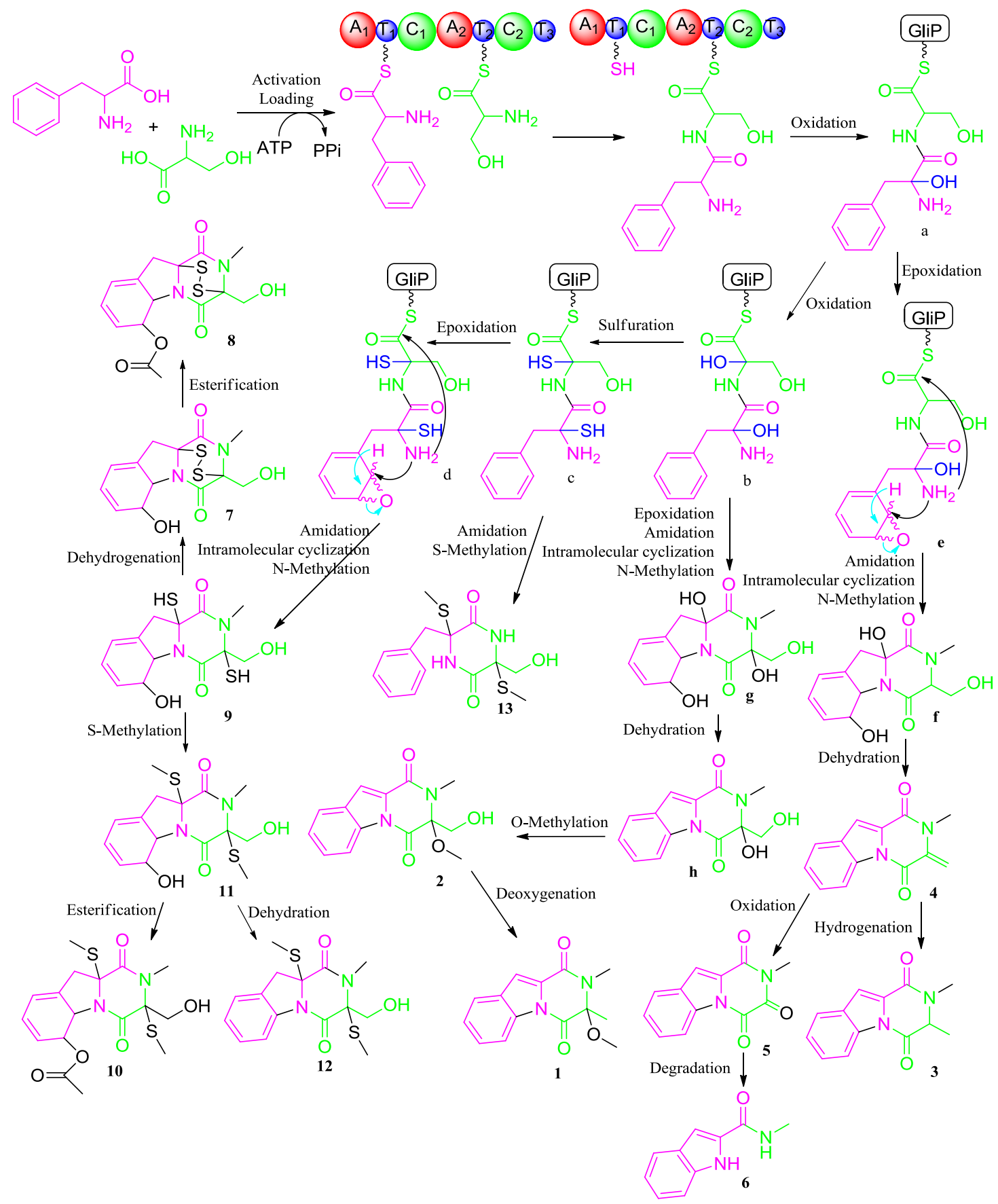


Compound 14 possessed a unique tetracyclic-fused skeleton, and it was the diastereomer of pseudofischerine [34]. We postulated that the biosynthetic pathway may involve D-valine, D-tryptophan and anthranilic acid as the precursors (Figure 5). Chaetominine [35] and kapakahines [36] have a similar tetracyclic-fused fragment.

Figure 5. Proposed biosynthetic pathway of compound 14.

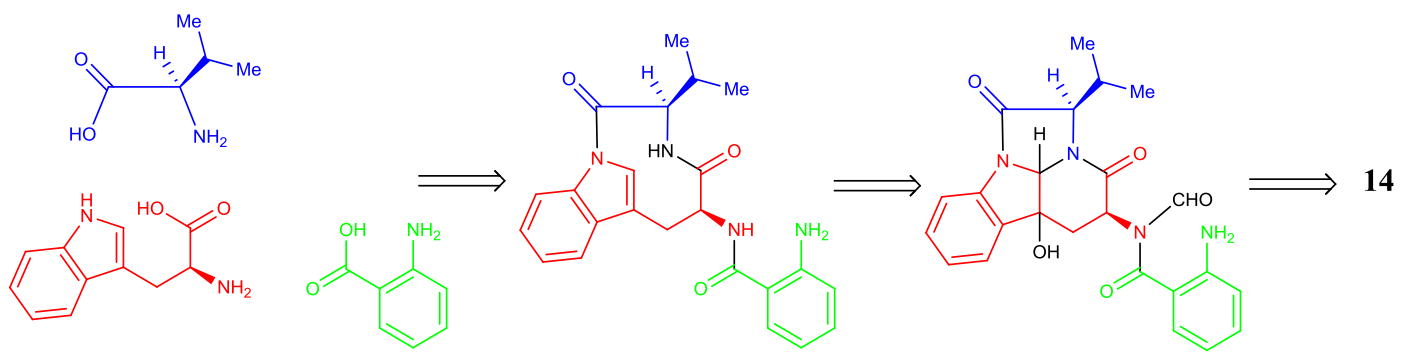

\subsection{Biological Activity}

Compounds 2-14 were evaluated for their antibacterial activity against three multidrug-resistant bacteria, i.e., the Gram-positive Staphylococcus aureus (ATCC29213) and Methicillin-resistant staphylococcus aureus (R3708) and the Gram-negative Escherichia coli (ATCC25922), using a broth dilution method (Mueller-Hinton broth) [37]. Vancomycin and ampicillin sodium were used as positive controls. Compounds 7 and 9 displayed significant inhibitory activities against these three bacteria with MIC values ranging from 1.52 to $97.56 \mu \mathrm{M}$ (Table 7). Compounds 4 and 8 inhibited the growth of Staphylococcus aureus ATCC29213 and R3708 with MIC values of 283.11, $70.70 \mu \mathrm{M}$ and 86.91, $21.73 \mu \mathrm{M}$, respectively. The remaining nine compounds, $\mathbf{2}, \mathbf{3}, \mathbf{5}, \mathbf{6}$ and $\mathbf{1 0}-\mathbf{1 4}$, were inactive in this assay ( $\mathrm{MIC}>256 \mu \mathrm{g} / \mathrm{mL}$ ). The results suggested that the bioactive compounds are more active against the Gram-positive bacteria. Especially, compounds 7 and 9 showed potent inhibition against Staphylococcus aureus R3708 with MIC values of 1.53 and $1.52 \mu \mathrm{M}$. Preliminary analysis of the structure-activity relationships of these twelve diketopiperazines suggests that the disulfide bridge or reduced disulfide bond is essential for the inhibitory activity. If the thiol groups are substituted, like compounds 10-13, the inhibitory effects disappeared. The substitution at the six-membered ring containing two conjugated double bonds influences the intensity of antibacterial activity. The analogues with a hydroxyl group at C-6 enhance the antibacterial activity compared to the analogues with an acetyl group at the same position. Additionally, the $\alpha$-methylene ketone group is also the pharmacophore for the antibacterial activity.

Table 7. Antibacterial activities of diketopiperazines 4 and 7-9 (MIC, $\mu \mathrm{M}, n=3)$.

\begin{tabular}{cccc}
\hline Compound & $\begin{array}{c}\text { Staphylococcus } \\
\text { aureus }(\mathbf{A T C C 2 9 2 1 3 )})\end{array}$ & $\begin{array}{c}\text { Staphylococcus } \\
\text { aureus }(\mathbf{R 3 7 0 8})\end{array}$ & $\begin{array}{c}\text { Escherichia coli } \\
\text { (ATCC25922) }\end{array}$ \\
\hline $\mathbf{4}$ & 283.11 & 70.70 & $>1,132$ \\
$\mathbf{7}$ & 12.20 & 1.53 & 24.53 \\
$\mathbf{8}$ & 86.91 & 21.73 & $>695.65$ \\
$\mathbf{9}$ & 48.78 & 1.52 & 97.56 \\
Vancomycin & 0.84 & 2.01 & \\
Ampicillin sodium & 8.07 & 129.24 & 6.73 \\
\hline
\end{tabular}


Furthermore, compounds 4, 5 and 7-13 were screened for their cytotoxic activities on the human embryonic kidney (HEK) 293 cell line and human colon cancer cell lines, HCT-116 and RKO (a poorly differentiated colon carcinoma cell line). Compounds 4, 7-9 and $\mathbf{1 1}$ exhibited potent cytotoxicities against these cell lines (Table 8). With a disulfide bridge in the molecule, compounds 7 and 8 showed potent cytotoxic activities. Compound $\mathbf{4}$ showed stronger inhibitory activities than compound 5; their structural difference is a typical $\alpha$-methylene ketone group in $\mathbf{4}$, whereas a diketone in 5. Compared to compounds 9 and 11, compound 10 lacked any activity ( $\mathrm{IC}_{50}>50 \mu \mathrm{M}$ ), supposedly since the thiol groups at C-3 and C-10a were methylated and the 6-OH was acetylated. The cytotoxic activities of the other compounds, due to the limited sample amount, were not tested in this assay.

Table 8. Cytotoxicities of compounds 4, 5 and $\mathbf{7 - 1 3}\left(\mathrm{IC}_{50}, \mu \mathrm{M}, n=5\right)$.

\begin{tabular}{cccc}
\hline \multirow{2}{*}{ Compound } & \multicolumn{3}{c}{ Cell line } \\
\cline { 2 - 4 } & 293 & HCT-116 & RKO \\
\hline 4 & $30.10 \pm 0.90$ & $10.34 \pm 1.41$ & $33.56 \pm 1.22$ \\
5 & $>50$ & $>50$ & $>50$ \\
7 & $1.58 \pm 0.03$ & $1.24 \pm 0.38$ & $0.80 \pm 0.20$ \\
8 & $4.49 \pm 0.24$ & $0.89 \pm 0.04$ & $1.24 \pm 0.18$ \\
9 & $1.26 \pm 0.04$ & $0.43 \pm 0.04$ & $0.41 \pm 0.07$ \\
10 & $>50$ & $>50$ & $>50$ \\
11 & $16.39 \pm 0.38$ & $8.59 \pm 0.96$ & $10.32 \pm 0.04$ \\
12 & $>50$ & $>50$ & $>50$ \\
13 & $>50$ & $>50$ & $>50$ \\
5 -Fluorouracil & & $2.04 \pm 0.22$ & $45.86 \pm 4.58$ \\
\hline
\end{tabular}

\section{Experimental Section}

\subsection{General Experimental Procedures}

Preparative HPLC was performed using a Shimadzu LC-20AT HPLC pump (Shimadzu Corporation, Nakagyo-ku, Kyoto, Japan) equipped with an SPD-20A dual $\lambda$ absorbance detector (Shimadzu Corporation, Nakagyo-ku, Kyoto, Japan) and a Shim-pack PRC-ODS HPLC column $(250 \times 20 \mathrm{~mm}$, Shimadzu Corporation, Nakagyo-ku, Kyoto, Japan). Optical rotations were measured using a Schmidt and Haensch Polartronic HNQW5 optical rotation spectrometer (SCHMIDT + HAENSCH GmbH \& Co., Berlin, Germany). CD spectra were measured on a JASCO J-810 circular dichroism spectrometer (JASCO International Co. Ltd., Hachioji, Tokyo, Japan). UV spectra were recorded on a Shimadzu UV-Vis-NIR spectrophotometer (Shimadzu Corporation, Nakagyo-ku, Kyoto, Japan). 1D and 2D NMR spectra were recorded on Bruker Avance IIIT 600HD and Bruker Avance II 400 spectrometers (Bruker BioSpin AG, Industriestrasse 26, Fällanden, Switzerland) and a Varian Mercury-Plus 300 spectrometer (Varian Medical Systems In., Salt Lake City, UT, USA). The chemical shifts are relative to the residual solvent signals $\left(\mathrm{CDCl}_{3}: \delta_{\mathrm{H}} 7.26\right.$ and $\delta_{\mathrm{C}} 77.0$; DMSO- $d_{6}: \delta_{\mathrm{H}} 2.50$ and $\delta_{\mathrm{C}} 39.51$; acetone- $d_{6}: \delta_{\mathrm{H}} 2.05$ and $\left.\delta_{\mathrm{C}} 29.92\right)$. Mass spectra were obtained on Thermo DSQ EI low-resolution and Thermo MAT95XP EI high-resolution mass spectrometers (Thermo Fisher Scientific In., Waltham, MA, USA). 


\subsection{Fungal Strain and Culture Method}

The marine fungus, Neosartorya pseudofischeri (Collection No. 2014F27-1), was isolated from the inner tissue of the sea star, Acanthaster planci, collected from Hainan Sanya National Coral Reef Reserve, China. This fungal strain was maintained in $15 \%(\mathrm{v} / \mathrm{v})$ glycerol aqueous solution at $-80{ }^{\circ} \mathrm{C}$. A voucher specimen was deposited in the School of Pharmaceutical Sciences, Sun Yat-sen University, Guangzhou, China. Analysis of the ITS rDNA (GenBank KF999816) by BLAST database screening provided a $100 \%$ match to $N$. pseudofischeri.

This fungus were cultured in GlyPY medium (glycerol $10 \mathrm{~g}$, peptone $5 \mathrm{~g}$, yeast extract $2 \mathrm{~g}, \mathrm{CaCO}_{3}$ $1 \mathrm{~g}$, sea water $1 \mathrm{~L}$ ) and GluPY medium (glucose $10 \mathrm{~g}$, peptone $5 \mathrm{~g}$, yeast extract $2 \mathrm{~g}$, sea water $1 \mathrm{~L}$, $\mathrm{pH}$ 7.5), respectively. Fungal mycelia were cut and transferred aseptically to $500 \mathrm{~mL}$ Erlenmeyer flasks each containing $200 \mathrm{~mL}$ sterilized GlyPY or GluPY liquid media. The flasks were incubated at $28^{\circ} \mathrm{C}$ on a rotary shaker $(120 \mathrm{rpm})$ for 25 days.

\subsection{Extraction and Isolation}

Fifty liters of GlyPY growth culture broth were filtered through cheesecloth. The culture broth was successively extracted with EtOAc $(50$ L) three times. The EtOAc extract was concentrated by low-temperature rotary evaporation. The extract ( $9.8 \mathrm{~g}$ ) was chromatographed on a silica gel column (diameter: $8 \mathrm{~cm}$, length: $70 \mathrm{~cm}$, silica gel, $200 \mathrm{~g}$ ) using petroleum ether $(2 \mathrm{~L})$, EtOAc (2 liters) $(100: 0-0: 100, \mathrm{v} / \mathrm{v})$, followed by EtOAc $(2 \mathrm{~L})$ and $\mathrm{MeOH}(2 \mathrm{~L})(100: 0-0: 100, \mathrm{v} / \mathrm{v})$ as the eluent to afford 10 fractions (code Fraction 1-Fraction 10). Fraction 2 was purified by the recrystallization in the petroleum ether-EtOAc $(3: 1, \mathrm{v} / \mathrm{v})$ solution to give compounds $4(13.4 \mathrm{mg})$ and $5(52.7 \mathrm{mg})$. Fraction 3 was separated with a preparative RP HPLC using a gradient elution $\mathrm{MeOH}-\mathrm{H}_{2} \mathrm{O}(20: 80$ up to $100: 0, \mathrm{v} / \mathrm{v}$ ) and then on Sephadex LH-20 using $\mathrm{MeOH}$ as the eluent followed by preparative RP HPLC eluted with $\mathrm{MeOH}-\mathrm{H}_{2} \mathrm{O}(70: 30, \mathrm{v} / \mathrm{v})$ to give compounds $1(1.3 \mathrm{mg}), 2$ (2.2 mg), 3 (1.62 mg) and $12(2.15 \mathrm{mg})$. Fraction 5 was purified with a preparative RP HPLC $\left(\mathrm{MeOH}-\mathrm{H}_{2} \mathrm{O}, 65: 35\right.$, v/v) to give compound $6(2.3 \mathrm{mg})$. Compound $10(7.2 \mathrm{mg})$ was obtained from Fraction 7 with a preparative RP HPLC (MeOH- $\left.\mathrm{H}_{2} \mathrm{O}, 70: 30, \mathrm{v} / \mathrm{v}\right)$.

Fifty liters of GluPY growth culture broth were filtered through cheesecloth. The culture broth was successively extracted with EtOAc $(50 \mathrm{~L})$ three times to afford $10.2 \mathrm{~g}$ of extract. The crude extract was separated as ten fractions (Fraction 1-10) by a silica gel column chromatograph (diameter: $8 \mathrm{~cm}$; length: $70 \mathrm{~cm}$; silica gel, $200 \mathrm{~g}$ ) employing the gradient elution described above. Fraction 3 displayed interesting signals in the $\delta_{\mathrm{H}} 8 \sim 9$ region and was further purified by RP HPLC using $\mathrm{MeCN}-\mathrm{H}_{2} \mathrm{O}$ $(40: 60, \mathrm{v} / \mathrm{v})$ as the eluent to yield compounds $14(3.2 \mathrm{mg})$ and $\mathbf{1 5}(4.0 \mathrm{mg})$. Fraction 4 was separated on a silica gel column chromatograph (diameter: $3 \mathrm{~cm}$; length: $50 \mathrm{~cm}$; silica gel, $50 \mathrm{~g}$ ) using an elution with petroleum ether $(300 \mathrm{~mL})$ and EtOAc $(300 \mathrm{~mL})(50: 50, \mathrm{v} / \mathrm{v})$ to obtain five fractions (Fractions 4-1 to Fractions 4-5). Fractions 4-2 was further purified on preparative RP HPLC eluted with $\mathrm{MeOH}-\mathrm{H}_{2} \mathrm{O}(75: 25, \mathrm{v} / \mathrm{v})$ to give compound $7(32 \mathrm{mg})$, under the same experiment condition. Fraction 4-4 afforded compounds $\mathbf{1 0}(10.2 \mathrm{mg}), \mathbf{1 1}(71.3 \mathrm{mg})$ and $\mathbf{1 3}(23.1 \mathrm{mg})$. Fraction 7 was purified on RP HPLC (MeOH- $\left.\mathrm{H}_{2} \mathrm{O}, 60: 40, \mathrm{v} / \mathrm{v}\right)$ to give compounds $8(7.5 \mathrm{mg})$ and $9(9.3 \mathrm{mg})$.

Neosartin A (1): Yellowish solid. UV (MeOH) $\lambda_{\max }(\varepsilon) 211(25,800), 243(30,637), 295$ $(21,930) \mathrm{nm}$. IR: $v_{\max } 3000,2926,1716,1656,1589,1576,1427,1391,1357,1228,1112,1043,844$, 
$750 \mathrm{~cm}^{-1} .{ }^{1} \mathrm{H}$ and ${ }^{13} \mathrm{C}$ NMR: Table 1. LREIMS: $m / z$ 256, 243, 227, 215, 199, 187, 170, 156, 143, 129 , 115, 103, 92, 89, 78, 72, 63, 56. HREIMS: $m / z$ [M] $]^{+}$calcd. for $\mathrm{C}_{14} \mathrm{H}_{14} \mathrm{O}_{3} \mathrm{~N}_{2}: 258.0999$; found 258.0998.

Neosartin B (2): Yellowish solid. UV (MeOH) $\lambda_{\max }(\varepsilon) 212$ (17,237), 243 (21,941), 295 (16,195) nm. IR: $v_{\max } 3301,3126,2951,1706,1635,1591,1577,1435,1392,1360,1348,1224$, 1151, 1116, 1081, 981, 746, $733 \mathrm{~cm}^{-1} .{ }^{1} \mathrm{H}$ and ${ }^{13} \mathrm{C}$ NMR: Table 1 . LREIMS: $m / z$ 274, 260, 243, 229, 215, 202, 188, 172, 156, 143, 130, 115, 103, 89, 83, 72, 57. HREIMS: $m / z[\mathrm{M}]^{+}$calcd. for $\mathrm{C}_{14} \mathrm{H}_{14} \mathrm{O}_{4} \mathrm{~N}_{2}$ : 274.0948; found 274.0947.

1,2,3,4-Tetrahydro-2,3-dimethyl-1,4-dioxopyrazino[1,2-a]indole (3): White solid. ${ }^{1} \mathrm{H}$ and ${ }^{13} \mathrm{C}$ NMR: Table 1. LREIMS: $m / z$ 228, 213, 200, 185, 172, 143, 131, 115, 100, 89, 71, 62, 56.

1,2,3,4-Tetrahydro-2-methyl-3-methylene-1,4-dioxopyrazino[1,2-a]indole (4): White solid. ${ }^{1} \mathrm{H}$ and ${ }^{13}$ C NMR: Table 2. LREIMS: $m / z$ 226, 199, 185, 169, 157, 143, 129, 115, 99, 88, 75, 62, 55.

1,2,3,4-Tetrahydro-2-methyl-1,3,4-trioxopyrazino[1,2-a]indole (5): White solid. ${ }^{1} \mathrm{H}$ and ${ }^{13} \mathrm{C}$ NMR: Table 2. LREIMS: $\mathrm{m} / z$ 228, 200, 159, 143, 131, 115, 100, 88, 71, 62, 50.

$\mathrm{N}$-methyl-1H-indole-2-carboxamide (6): White solid. ${ }^{1} \mathrm{H}$ and ${ }^{13} \mathrm{C}$ NMR: Table 2. LREIMS: $\mathrm{m} / z$ 174, $156,143,115,89,77,63,58$.

Gliotoxin (7): Pale yellowish solid. ${ }^{1} \mathrm{H}$ NMR $\left(\mathrm{CDCl}_{3}, 400 \mathrm{MHz}\right): 5.98$ (1H, d, 4.0, H-7), $5.92(1 \mathrm{H}$, dd, 9.2, 4.2, H-8), 5.84 (1H, s, OH-13), 5.75 (1H, d, 9.2, H-9), 4.80 (2H, s, H-5a, H-6), 4.43 (1H, d, 17.6, H-12), 4.23 (1H, d, 17.6, H-12), $4.06(1 \mathrm{H}, \mathrm{brs}, \mathrm{OH}-12), 3.73(1 \mathrm{H}, \mathrm{d}, 12.8, \mathrm{H}-10), 3.19$ (3H, s, $\left.\mathrm{CH}_{3}-11\right), 2.94$ (1H, d, 12,8, H-10); ${ }^{13} \mathrm{C}\left(\mathrm{CDCl}_{3}, 100 \mathrm{MHz}\right): 166.0$ (C-1), 165.1 (C-4), 130.8 (C-9a), 129.8 (C-7), 123.3 (C-8), 120.1 (C-9), 77.3 (C-3), 75.8 (C-10a), 73.1 (C-6), 69.7 (C-5a), 60.4 (C-12), 36.5 (C-10), 27.5 (C-11). LREIMS: $m / z$ 326, 308, 277, 262, 244, 233, 217, 199, 188, 160, 144, 132 , $107,89,77,73,64,55,42$.

Acetylgliotoxin (8): Yellowish solid. ${ }^{1} \mathrm{H}$ and ${ }^{13} \mathrm{C}$ NMR: Table 3.

Reduced gliotoxin (9): Yellowish solid. ${ }^{1} \mathrm{H}$ and ${ }^{13} \mathrm{C}$ NMR: Table 3.

6-Acetylbis(methylthio)gliotoxin (10): Yellowish oil. ${ }^{1} \mathrm{H}$ NMR $\left(\mathrm{CDCl}_{3}, 400 \mathrm{MHz}\right): 6.16(1 \mathrm{H}, \mathrm{d}, 14.8$, H-7), 5.90 (2H, m, H-8, H-9), 5.75 (1H, d, 10.4, H-6), 5.01 (1H, d, 14.4, H-5a), 4.23 (1H, d, 11.2, H-12), $3.76(1 \mathrm{H}, \mathrm{d}, 11.6, \mathrm{H}-12), 3.60(1 \mathrm{H}, \mathrm{brs}, \mathrm{OH}-12), 3.09\left(3 \mathrm{H}, \mathrm{s}, \mathrm{CH}_{3}\right), 3.04(1 \mathrm{H}, \mathrm{d}, 16.0, \mathrm{H}-10), 2.90(1 \mathrm{H}$, d, 16.0, H-10), 2.29 (3H, s, CH3-13), $2.12\left(3 \mathrm{H}, \mathrm{s}, \mathrm{CH}_{3}-15\right), 2.05\left(3 \mathrm{H}, \mathrm{s}, \mathrm{COCH}_{3}-14\right) ;{ }^{13} \mathrm{C}\left(\mathrm{CDCl}_{3}, 100\right.$ MHz): $170.7\left(\mathrm{COCH}_{3}-14\right), 166.4$ (C-1), 164.4 (C-4), 133.8 (C-9a), 127.8 (C-7), 123.3 (C-8), 119.8 (C-9), 72.7 (C-3), 72.3 (C-10a), 75.2 (C-6), 65.4 (C-5a), 63.6 (C-12), 40.1 (C-10), 28.7 (C-11), 21.3 ( $\left.\mathrm{COCH}_{3}-14\right), 15.0(\mathrm{C}-13), 13.0\left(\mathrm{CH}_{3}-15\right)$.

Bisdethiobis(methylthio)gliotoxin (11): Yellowish oil. ${ }^{1} \mathrm{H} \mathrm{NMR}\left(\mathrm{CDCl}_{3}, 400 \mathrm{MHz}\right): 5.85(2 \mathrm{H}, \mathrm{m}$, H-7, H-8), 5.65 (1H, d, 9.2, H-9), 4.86 (2H, s, H-5a, H-6), 4.30 (1H, d, 11.6, H-12), 3.82 (1H, d, 11.6, $\mathrm{H}-12), 3.09$ (3H, s, $\left.\mathrm{CH}_{3}-11\right), 3.01$ (1H, d, 16.0, H-10), 2.92 (1H, d, 16.0, H-10), 2.22 (3H, s, $\left.\mathrm{CH}_{3}-13\right)$, 2.19 (3H, s, $\left.\mathrm{CH}_{3}-15\right) ;{ }^{13} \mathrm{C}\left(\mathrm{CDCl}_{3}, 100 \mathrm{MHz}\right): 166.7$ (C-1), 165.9 (C-4), 131.7 (C-9a), 129.5 (C-7), 123.2 (C-8), 119.8 (C-9), 74.2 (C-3), 72.1 (C-10a), 71.4 (C-6), 69.4 (C-5a), 63.4 (C-12), 38.7 (C-10), $28.5\left(\mathrm{CH}_{3}-11\right), 15.0\left(\mathrm{CH}_{3}-13\right), 13.0\left(\mathrm{CH}_{3}-15\right)$.

Didehydrobisdethiobis(methylthio)gliotoxin (12): Yellowish oil. ${ }^{1} \mathrm{H}$ and ${ }^{13} \mathrm{C}$ NMR: Table 4. 
Bis- $N$-norgliovictin (13): White solid. ${ }^{1} \mathrm{H}$ and ${ }^{13} \mathrm{C}$ NMR: Table 4.

Neosartin C (14): Yellowish solid. $[\alpha]_{D}^{20}:-58.17^{\circ}(c=0.0822, \mathrm{MeOH})$. UV (MeOH) $\lambda_{\max }(\varepsilon) 314$ (2910), 301 (3653), 226 (35,975), 212 (29,412) nm. IR: $v_{\max }$ 3348, 3068, 2970, 2934, 2875, 1724, 1667, 1608, 1476, 1465, 1387, 1326, 1292, 1267, 1248, 1172, 1112, 1076, 1007, 976, 915, 891, 844, 764, 742, 700, 593, $560 \mathrm{~cm}^{-1} .{ }^{1} \mathrm{H}$ and ${ }^{13} \mathrm{C}$ NMR: Table 5. LREIMS: $\mathrm{m} / z$ 430, 412, 341, 329, 314, 298, 286, 266, 238, 224, 184, 147, 130, 102, 83, 76, 55. HREIMS: $m / z[\mathrm{M}]^{+}$calcd. for $\mathrm{C}_{24} \mathrm{H}_{22} \mathrm{O}_{4} \mathrm{~N}_{4}$ : 430.1636 ; found 430.1635 .

Pyripyropene A (15): Yellowish solid. UV (MeOH) $\lambda_{\max }(\varepsilon) 320$ (18,029), $231(29,463), 209$ $(15,006) \mathrm{nm}$. IR: $v_{\max } 3409,2975,2947,2883,1723,1644,1580,1481,1436,1394,1370,1234,1159$, 1109, 1090, 1074, 1040, 1026, 1009, 984, 960, 925, 873, 809, 763, 704, 687, 650, 603, $583 \mathrm{~cm}^{-1} .{ }^{1} \mathrm{H}$ and ${ }^{13} \mathrm{C}$ NMR: Table 6. LREIMS: $m / z$ 583, 565, 550, 523, 505, 494, 463, 445, 430, 403, 385, 358, 334, 304, 283, 240, 218, 202, 190, 171, 157, 148, 133, 119, 106, 95, 79, 69, 55.

\subsection{Antibacterial Activity Assay}

The MIC values were determined using a broth dilution method (Mueller-Hinton broth) based on the National Committee for Clinical Laboratory Standards (NCCLS) standard. The starting concentrations of the tested compounds were $256 \mu \mathrm{g} / \mathrm{mL}$ (from 256 to 0.25 ). The solution of compound in DMSO $(10 \mu \mathrm{L})$ was added to $90 \mu \mathrm{L}$ of bacterial culture $\left(1 \times 10^{6} \mathrm{CFU} / \mathrm{mL}\right)$ in the first well of flat-bottomed 96-well tissue culture plates. The solution was then double diluted. The bacterial culture solution containing the appropriate compound $(50 \mu \mathrm{L})$ was discarded from the last well in order to ensure a $100-\mu \mathrm{L}$ volume of bacterial culture in every well. A set of tubes containing only inoculated broth and solvent were kept as controls. The plate was incubated at $37{ }^{\circ} \mathrm{C}$ overnight in an electroheating standing-temperature cultivator before the measurement of the absorbance value. The optical density values at $600 \mathrm{~nm}$ were measured using a multifunction microplate reader (PowerWaveTM XS2, BioTek ${ }^{\circledR}$ Instruments Inc., Winooski, VT, USA). Vancomycin and ampicillin sodium were used as positive controls.

\subsection{Cytotoxicity Assay}

Compounds in DMSO at $50 \mathrm{mM}$ were used in the MTS ((3-(4,5-dimethylthiazol-2-yl)-5-(3-car boxymethoxyphenyl)-2-(4-sulfophenyl)-2 $H$-tetrazolium)) assay. 5-Fluorouracil was used as the positive control, and DMSO was used as the negative control. 293, HCT116 and RKO cells were suspended in fresh RPMI-1640 medium containing 10\% fetal bovine serum and $100 \mu \mathrm{g} / \mathrm{mL}$ penicillin and streptomycin at a cell density of $1 \times 10^{5}$ cells $/ \mathrm{mL}$ and seeded into 96 -well plates each $100 \mu \mathrm{L} /$ well. The suspension cells, 293, HCT116 and RKO cells, were incubated at $37{ }^{\circ} \mathrm{C}$ for $12 \mathrm{~h}$. Then, compounds were added to the cultures at different concentrations; then, the cells were cultured at $37{ }^{\circ} \mathrm{C}$ for $72 \mathrm{~h}$. Twenty microliters of MTS/PMS (phenazine methosulfate) were added into each well, incubated at $37{ }^{\circ} \mathrm{C}$ for $4 \mathrm{~h}$ in a humidified, $5 \% \mathrm{CO}_{2}$ atmosphere. The absorbance at $490 \mathrm{~nm}$ was recorded using a Thermo Scientific Varioskan Flash Multimode Reader (Thermo Fisher Scientific Inc., Waltham, MA, USA). The data were analyzed with the GraphPad Prism 6 software package [38]. 


\section{Conclusions}

Alternating the cultivation parameters systematically, such as the components of the media, to enhance the diversity of secondary metabolites produced by the fungus is a well-known, simple and efficient technique. Using this strategy, we isolated and identified fifteen compounds, including twelve diketopiperazine derivatives, a meroterpenoid, an alkaloid with a unique tetracyclic-fused skeleton and an imidazole analogue from the two cultures. The discovery of these compounds provided further evidence that the genus of Neosartorya is a rich source of nitrogen-containing natural products. Interestingly, the dominant metabolites from the GluPY medium were the diketopiperazines with disulfide bonds; however, the main compounds from the GlyPY medium were the diketopiperazines without a disulfide bond. The biosynthetic pathways of the unique alkaloids are complex and diverse. Hopefully, further investigation on the secondary metabolites of Neosartorya pseudofischeri in varied culture conditions supplied with amino acid precursors may find more novel alkaloids and improve their production. Most of the metabolites showed significant antibacterial and cytotoxic activities. Based on the structure-activity relationship analysis, the disulfide bridge, the $\alpha$-methylene ketone group, the hydroxyl group at C-6 and the thiol groups were considered as the pharmacophores.

\section{Acknowledgments}

This project was financially supported by the National Natural Science Foundation of China (Nos. 30973633 and 81102502), the Special Financial Fund of Innovative Development of the Marine Economic Demonstration Project (GD2012-D01-001), the Guangdong Provincial Science and Technology Research Program (Nos. 2012A031100005, 2013B021100010 and 2013B021100012), the Guangdong Natural Science Foundation (No. S2012010010653), and the Guangzhou Science and Technology Research Program (No. 2014J4100059).

\section{Author Contributions}

Conceived of and designed the experiments: Wen-Jian Lan, Hou-Jin Li. Performed the experiments: Wan-Ling Liang, Xiu Le, Xiang-Ling Yang, Jun-Xiong Chen, Huan-Liang Liu, Lai-You Wang, Kun-Teng Wang, Kun-Chao Hu, De-Po Yang. Wrote the paper: Wan-Ling Liang, Wen-Jian Lan, Hou-Jin Li, Jun Xu.

\section{Conflicts of Interest}

The authors declare no conflict of interest.

\section{References}

1. Wang, Y.; Zheng, J.K.; Liu, P.P.; Wang, W.; Zhu, W.M. Three new compounds from Aspergillus terreus PT06-2 grown in a high salt medium. Mar. Drugs 2011, 9, 1368-1378.

2. Wang, W.J.; Li, D.Y.; Hua, H.M.; Ma, E.L.; Li, Z.L. Caryophyllene sesquiterpenes from the marine-derived fungus Ascotricha sp. ZJ-M-5 by the one strain-many compounds strategy. J. Nat.

Prod. 2014, 77, 1367-1371. 
3. James, W.; Suzanne, M.S.; David, J.N.; Karina, M.Z. Tetramic acid analogues produced by coculture of Saccharopolyspora erythraea with Fusarium pallidoroseum. J. Nat. Prod. 2014, 77, 173-177.

4. Samuel, B.; Olivier, S.; Nadine, B.; Michel, M.; Katia, G.; Jean-Luc, W. De novo production of metabolites by fungal co-culture of Trichophyton rubrum and Bionectria ochroleuca. J. Nat. Prod. 2013, 76, 1157-1165.

5. Wang, F.Z.; Wei, H.J.; Zhu, T.J.; Li, D.H.; Lin, Z.J.; Gu, Q.Q. Three new cytochalasins from the marine-derived fungus Spicaria elegans KLA03 by supplementing the cultures with L- and D-Tryptophan. Chem. Biodivers. 2011, 8, 887-894.

6. Christopher, T.W.; Stuart, W.H.; Brian, D.A.; Gao, X.; Tang, Y. Short pathways to complexity generation: Fungal peptidyl alkaloid multicyclic scaffolds from anthranilate building blocks. ACS Chem. Biol. 2013, 8, 1366-1382.

7. Jeremy, B.; Nida, M.; Whittney, N.B.; Lacey, H.; Lindsey, N.S.; Tina, M.; Dennis, E.K.; Betty, B.; Alberto, van O.; Bill, B. Epigenetic tailoring for the production of anti-infective cytosporones from the marine fungus Leucostoma persoonii. Mar. Drugs 2012, 10, 762-774.

8. Teigo, A.; Takashi, Y.; Yoshiteru, O. Aromatic polyketide productionin cordyceps indigotica, an entomopathogenic fungus, induced by exposure to a histone deacetylase inhibitor. Org. Lett. 2012, 14, 2006-2009.

9. Li, H.J.; Jiang, W.H.; Liang, W.L.; Huang, J.X.; Mo, Y.F.; Ding, Y.Q.; Lam, C.K.; Qian, X.J.; Zhu, X.F.; Lan, W.J. Induced marine fungus Chondrostereum sp. as a means of producing new sesquiterpenoids chondrosterins I and J by using glycerol as the carbon source. Mar. Drugs 2014, 12, 167-175.

10. Li, H.J.; Chen, T.; Xie, Y.L.; Chen, W.D.; Zhu, X.F.; Lan, W.J. Isolation and structural elucidation of chondrosterins F-H from the marine fungus Chondrostereum sp. Mar. Drugs 2013, $11,551-558$.

11. Li, H.J.; Xie, Y.L.; Xie, Z.L.; Chen, Y.; Lam, C.K.; Lan, W.J. Chondrosterins A-E, triquinane-type sesquiterpenoids from soft coral-associated fungus Chondrostereum sp. Mar. Drugs 2012, 10, 627-638.

12. Li, H.J.; Lan, W.J.; Lam, C.K.; Yang, F.; Zhu, X.F. Hirsutane sesquiterpenoids from the marine- derived fungus Chondrostereum sp. Chem. Biodivers. 2011, 8, 317-324.

13. Lan, W.J.; Zhao, Y.; Xie, Z.L.; Liang, L.Z.; Shao, W.Y.; Zhu, L.P.; Yang, D.P.; Zhu, X.F.; Li, H.J. Novel sorbicillin analogues from the marine fungus Trichoderma sp. associated with the seastar Acanthaster planci. Nat. Prod. Commun. 2012, 7, 1337-1340.

14. Zhao, Y.; Li, S.Q.; Li, H.J.; Lan, W.J. Lanostane triterpenoids from the fungus Ceriporia lacerate. Chem. Nat. Compd. 2013, 49, 653-656.

15. Xie, Z.L.; Li, H.J.; Wang, L.Y.; Liang, W.L.; Liu, W.; Lan, W.J. Trichodermaerin, a new lactone from the marine fungus Trichoderma erinaceum associated with the sea star Acanthaster planci. Nat. Prod. Commun. 2013, 8, 67-68.

16. Lan, W.J.; Liu, W.; Liang, W.L.; Xu, Z.; Le, X.; Xu, J.; Lam, C.K.; Yang, D.P.; Li, H.J.; Wang, L.Y. Pseudaboydins A and B: Novel isobenzofuranone derivatives from marine fungus Pseudallescheria boydii associated with starfish Acanthaster planci. Mar. Drugs 2014, 12, 4188-4199. 
17. Peterson, S.W. Neosartorya pseudofischeri sp. nov. and its relationship to other species in Aspergillus section Fumigati. Mycol. Res. 1992, 96, 547-554.

18. Yukihiro, A.; Hideaki, K.; Rie, O.; Arika, Y.; Hiroshi, M.; Hiroyuki, O. Azaspirene: A novel angiogenesis inhibitor containing a 1-oxa-7-azaspiro[4.4]non-2-ene-4,6-dione skeleton produced by the fungus Neosartorya sp. Org. Lett. 2002, 4, 2845-2848.

19. Marco, M.; Anna, A.; Veronique, M.; Angela, B.; Alessio, C.; Laetitia, M.Y.B.; Maurizio, V.; Alexander, K.; Robert, K.; Antonio, E. Fischerindoline, a pyrroloindole sesquiterpenoid isolated from Neosartorya pseudofischeri, with in vitro growth inhibitory activity in human cancer cell lines. Tetrahedron 2013, 69, 7466-7470.

20. John, R.J.; James, B.B. Gliotoxin. X. dethiogliotoxin and related compounds. J. Am. Chem. Soc. 1953, 75, 2103-2109.

21. Ajay, K.B.; Das, K.G.; Funke, P.T.; Irene, K.; Shukla, O.P.; Khanchandani, K.S.; Suhadolnik, R.J. Biosynthetic studies on gliotoxin using stable isotopes and mass spectral methods. J. Am. Chem. Soc. 1968, 90, 1038-1041.

22. Johnson, J.R.; Hasbrouck, R.B.; Dutcher, J.D.; Bruce, W.F. Gliotoxin. V. The Structure of certain indole derivatives related to gliotoxin. J. Am. Chem. Soc. 1945, 67, 423.

23. Ali, M.S.; Shannon, J.S.; Taylor, A. Isolation and structures of 1,2,3,4-tetrahydro-1,4-di oxopyrazino[1,2-a]-indoles from cultures of Penicillium terlikowskii. J. Chem. Soc. C 1968, 2044-2048, doi: 10.1039/J39680002044.

24. Mourad, K. Gliotoxin: Uncommon ${ }^{1} \mathrm{H}$ couplings and revised ${ }^{1} \mathrm{H}$ and ${ }^{13} \mathrm{C}-\mathrm{NMR}$ assignments. J. Nat. Prod. 1990, 53, 717-719.

25. Katharine, R.W.; Joseline, R.; Ang, K.H.; Karen, T.; Jennifer, E.C.; James, M.; Phillip, C. Assessing the trypanocidal potential of natural and semi-synthetic diketopiperazines from two deep water marine-derived fungi. Bioorg. Med. Chem. 2010, 18, 2566-2574.

26. Didier, V.D.; Junji, I.; Kazuro, S.; Hong, Y.; Hideo, T.; Satoshi, O. Inhibition of farnesyl-protein transferase by gliotoxin and acetylgliotoxin. J. Antibiot. 1992, 45, 1802-1805.

27. Daniel, H.S.; Nicole, R.; Thorsten, H.; Peter, H.; Axel, A.B.; Christian, H. Transannular disulfide formation in gliotoxin biosynthesis and its role in self-resistance of the human pathogen Aspergillus fumigates. J. Am. Chem. Soc. 2010, 132, 10136-10141.

28. Afiyatullov, S.S.; Kalinovskii, A.I.; Pivkin, M.V.; Dmitrenok, P.S.; Kuznetsova, T.A. Alkaloids from the marine isolate of the fungus Aspergillus fumigatus. Chem. Nat. Compd. 2005, 41, 236-238.

29. Kirby, G.W.; Robins, D.J.; Sefton, M.A.; Talekar, R.R. Biosynthesis of bisdethiobis(methylthio) gliotoxin, a new metabolite of Gliocladium Deliquescens. J. Chem. Soc. Perkin Trans I. 1980, 119-121, doi:10.1039/P19800000119.

30. Zhao, W.Y.; Zhu, T.J.; Han, X.X.; Fan, G.T.; Liu, H.B.; Zhu, W.M.; Gu, Q.Q. A new gliotoxin analogue from a marine-derived fungus Aspergillus fumigatus Fres. Nat. Prod. Res. 2009, 23, 203-207.

31. Atsuki, O.; Kaoru, I.;Masaki, O.; Hiroshi, T.; Satoshi, O.; Tohru, N. Total synthesis of pyripyropene A. Tetrahedron 2011, 67, 8195-8203.

32. Mootz, H.D.; Marahiel, M.A. Biosynthetic systems for nonribosomal peptide antibiotic assembly. Curr. Opin. Chem. Biol. 1997, 1, 543-551. 
33. Balibar, C.J.; Walsh, C.T. GliP, a multimodular nonribosomal peptide synthetase in Aspergillus fumigatus, makes the diketopiperazine scaffold of gliotoxin. Biochemistry 2006, 45, 15029-15038.

34. Amnat, E.; Anake, K.; Céline, B.; Véronique, M.; Leka, M.; Florence, L.; Artur, S.; Robert, K.; Werner, H. Secondary metabolites from a culture of the fungus Neosartorya pseudofischeri and their In vitro activity in human cancer cells. Planta Med. 2012, 78, 1767-1776.

35. Jiao, R.H.; Xu, S.; Liu, J.Y.; Ge, H.M.; Ding, H.; Xu, C.; Zhu, H.L.; Tan, R.X. Chaetominine, a cytotoxic alkaloid produced by endophytic Chaetomium sp. IFB-E015. Org. Lett. 2006, 8, 5709-5712.

36. Bryan, K.S.; Yeung, Y.N.; Robin, B.K.; John, R.C.; Wesley, Y.Y.; Paul, J.S.; Michelle, K.B. The Kapakahines, cyclic peptides from the marine sponge Cribrochalina olemda. J. Org. Chem. 1996, $61,7168-7173$.

37. Casey, J.T.; O'Cleirigh, C.; Walsh, P.K.; O'Shea, D.G. Development of a robust microtiter plate-based assay method for assessment of bioactivity. J. Microbiol. Methods 2004, 58, 327-334.

38. GraphPad Prism 6; GraphPad Software, Inc.: La Jolla, CA, USA, 2014.

(C) 2014 by the authors; license MDPI, Basel, Switzerland. This article is an open access article distributed under the terms and conditions of the Creative Commons Attribution license (http://creativecommons.org/licenses/by/4.0/). 